\title{
CURVATURE-DIMENSION INEQUALITIES ON SUB-RIEMANNIAN MANIFOLDS OBTAINED FROM RIEMANNIAN FOLIATIONS, PART I
}

\author{
ERLEND GRONG \\ ANTON THALMAIER
}

\begin{abstract}
We give a generalized curvature-dimension inequality connecting the geometry of sub-Riemannian manifolds with the properties of its subLaplacian. This inequality is valid on a large class of sub-Riemannian manifolds obtained from Riemannian foliations. We give a geometric interpretation of the invariants involved in the inequality. Using this inequality, we obtain a lower bound for the eigenvalues of the sub-Laplacian. This inequality also lays the foundation for proving several powerful results in Part II.
\end{abstract}

\section{INTRODUCTION}

On a given connected manifold $M$, there is a well established relation between elliptic second order differential operators on $M$ and Riemannian geometries on the same space. More precisely, for any smooth elliptic operator $L$ on $M$ without constant term, there exist a unique Riemannian metric $\mathbf{g}$ on $M$ such that for any pair of smooth functions $f, g \in C^{\infty}(M)$,

$$
\Gamma(f, g):=\frac{1}{2}(L(f g)-f L g-g L f)=\langle\operatorname{grad} f, \operatorname{grad} g\rangle_{\mathbf{g}} .
$$

Conversely, from any Riemannian metric g, we can construct a second order operator satisfying (1.1) in the form of the Laplacian $\Delta$. In addition, the properties of $L$ and $\mathbf{g}$ are intimately connected. Consider the case when $M$ is complete with respect to $\mathbf{g}$ and write $P_{t}=e^{t / 2 \Delta}$ for the heat semigroup corresponding to $\frac{1}{2} \Delta$. Then the following statements are equivalent for any $\rho \in \mathbb{R}$.

(a) For any $f \in C_{c}^{\infty}(M), \rho\|\operatorname{grad} f\|_{\mathbf{g}}^{2} \leq \operatorname{Ric}_{\mathbf{g}}(\operatorname{grad} f$, $\operatorname{grad} f)$.

(b) For any $f \in C_{c}^{\infty}(M),\left\|\operatorname{grad} P_{t} f\right\|_{\mathbf{g}}^{2} \leq e^{-\rho t} P_{t}\|\operatorname{grad} f\|_{\mathbf{g}}^{2}$.

(c) For any $f \in C_{c}^{\infty}(M), \frac{1-e^{-\rho t}}{\rho}\left\|\operatorname{grad} P_{t} f\right\|_{\mathbf{g}}^{2} \leq P_{t} f^{2}-\left(P_{t} f\right)^{2}$.

Here, $C_{c}^{\infty}(M)$ is the space of smooth functions on $M$ with compact support and $\mathrm{Ric}_{\mathbf{g}}$ is the Ricci curvature tensor of $\mathbf{g}$. This equivalence gives us a way of understanding Ricci curvature in terms of growth of the gradient of a solution to the heat equation. With appropriate modifications of the Ricci curvature, the same statement holds for a general elliptic operator $L$ satisfying (1.1), giving us a geometric tool to study the heat flow of elliptic operators. See e.g. [26] and references therein for the full statement.

2010 Mathematics Subject Classification. 58J35 (53C17,58J99).

Key words and phrases. Hypoelliptic operators; Sub-Riemannian geometry; Curvaturedimension inequality; Spectral gap; Riemannian foliations. 
Let us now consider the case when $L$ is not elliptic, but is rather given locally in a form

$$
L=\sum_{i=1}^{n} A_{i}^{2}+\text { first order terms }
$$

Here, $A_{1}, \ldots, A_{n}$ are linearly independent vector fields and $n$ is strictly less than the dimension of $M$. In this case, there is still a geometry we can associate with $L$ by considering the subbundle spanned by $A_{1}, \ldots, A_{n}$ furnished with a metric tensor that makes these vector fields orthogonal. Such a geometry is called subRiemannian geometry. A sub-Riemannian manifold is a connected manifold $M$ with a positive definite metric tensor $\mathbf{h}$ defined only on a subbundle $\mathcal{H}$ of the tangent bundle $T M$. If we assume that sections of $\mathcal{H}$ and their iterated Lie brackets span the entire tangent bundle, we obtain a metric space $\left(M, \mathrm{~d}_{c c}\right)$, where the distance between two points is defined by taking the infimum of the lengths of all curves tangent to $\mathcal{H}$ that connect these points.

In recent years, understanding how to define curvature in sub-Riemannian geometry has become an important question. One approach has been to introduce curvature by studying invariants of the flow of normal geodesics associated to the sub-Riemannian structure, see e.g. 27, 18, 1, 5]. The other approach explores the interaction of the sub-Riemannian gradient and second order operators of the form (1.2). We will follow the latter approach.

The equivalence of statements (a), (b) and (c) mentioned previously is rooted in the curvature-dimension inequality for Riemannian manifolds. In the notation of Bakry and Émery [3] this inequality is written as

$$
\Gamma_{2}(f) \geq \frac{1}{n}(L f)^{2}+\rho \Gamma(f), \quad f \in C^{\infty}(M) .
$$

Here, $n=\operatorname{dim} M, L=\Delta, \rho$ is a lower bound for the Ricci curvature and, for any pair of functions $f, g \in C^{\infty}(M)$,

$$
\begin{array}{rlrl}
\Gamma(f, g) & =\frac{1}{2}(L(f g)-f L g-g L f)=\langle\operatorname{grad} f, \operatorname{grad} g\rangle_{\mathbf{g}}, & & \Gamma(f)=\Gamma(f, f), \\
\Gamma_{2}(f, g) & =\frac{1}{2}(L \Gamma(f, g)-\Gamma(f, L g)-\Gamma(L f, g)), & \Gamma_{2}(f)=\Gamma_{2}(f, f) .
\end{array}
$$

Even in simple cases, this inequality fails in the sub-Riemannian setting. The following generalization has been suggested by F. Baudoin and N. Garofalo in 8 .

Let $\mathbf{h}$ be a sub-Riemannian metric defined on a subbundle $\mathcal{H}$ of $T M$. Let $L$ be any second order operator as in (1.2), i.e. locally given as a sum of squares of an orthonormal basis of $\mathcal{H}$ plus a first order term. We remark that, unlike the Laplace operator on a Riemannian manifold, the operator $L$ is not uniquely determined by $\mathbf{h}$ unless we add some additional structure such as a chosen preferred volume form on $M$. Define $\Gamma$ and $\Gamma_{2}$ as in (1.3) and (1.4). For any positive semi-definite section $\mathbf{v}^{*}$ of $\operatorname{Sym}^{2} T M$, define $\Gamma^{\mathbf{v}^{*}}(f, g)=\mathbf{v}^{*}(d f, d g)$ and $\Gamma^{\mathbf{v}^{*}}(f)=\Gamma^{\mathbf{v}^{*}}(f, f)$. Let $\Gamma_{2}^{\mathrm{v}^{*}}(f)$ be defined analogous to $\Gamma_{2}(f)$ in (1.4). Then $L$ is said to satisfy a generalized curvature-dimension inequality if we can choose $\mathbf{v}^{*}$ such that for every $\ell>0$,

$$
\Gamma_{2}(f)+\ell \Gamma_{2}^{\mathbf{v}^{*}}(f) \geq \frac{1}{n}(L f)^{2}+\left(\rho_{1}-\ell^{-1}\right) \Gamma(f)+\rho_{2} \Gamma^{\mathbf{v}^{*}}(f),
$$

for some $1 \leq n \leq \infty, \rho_{1} \in \mathbb{R}$ and $\rho_{2}>0$. Using this inequality, the authors were able to prove several results, such as gradient bounds, Li-Yau type inequality and 
a sub-Riemannian version of the Bonnet-Myers theorem. See also further results based of the same formalism in [6, 7, 10, 14].

So far, the examples of sub-Riemannian manifolds satisfying (1.5) all have a complement to $\mathcal{H}$ spanned by the sub-Riemannian analogue of Killing vector fields. We want to show that a further generalization of (1.5) holds for a larger class of sub-Riemannian manifolds. We also want to give an interpretation for the constants involved in the curvature-dimension inequality. Results following from this inequality are important for sub-Riemannian geometry, understanding solutions of the heat equation of operators $L$ described locally as in (1.2), along with the stochastic processes which have these operators as their infinitesimal generators.

In order to motivate our approach, let us first consider the following example. Let $\pi: M \rightarrow B$ be a submersion between two connected manifolds and let $\breve{\mathbf{g}}$ be a Riemannian metric on $B$. Let $\mathcal{V}=\operatorname{ker} \pi_{*}$ be the vertical bundle and let $\mathcal{H}$ be an Ehresmann connection on $\pi$, that is, a subbundle such that $T M=\mathcal{H} \oplus \mathcal{V}$. We then define a sub-Riemannian metric $\mathbf{h}$ on $M$ by pulling back the Riemannian metric, i.e. $\mathbf{h}=\left.\pi^{*} \breve{\mathbf{g}}\right|_{\mathcal{H}}$. In this case, we have two notions of curvature that could be expected to play a role for the inequality of type (1.5), namely the Ricci curvature of $B$ and the curvature of the Ehresmann connection $\mathcal{H}$ (see Section 3.1 for definition). After all, our sub-Riemannian structure is uniquely determined by a metric on $B$ and a choice of Ehresmann connection. For this reason, examples of this type should be helpful in providing a geometric understanding of curvature in sub-Riemannian geometry. However, we have to deal with the following two challenges.

(i) Even though the sub-Riemannian geometry on $M$ can be considered as "lifted" from $B$ the same cannot be said for our operator $L$. That is, if $L$ is of the type (1.2), $\breve{\Delta}$ is the Laplacian on $B$ and $f \in C^{\infty}(B)$ is a smooth function on $B$, then $L(f \circ \pi)$ does not coincide with $(\check{\Delta} f) \circ \pi$ in general.

(ii) The same sub-Riemannian structure on $M$ can sometimes be considered as lifted from two different Riemannian manifolds (see Section 4.5 for an example).

Our approach to overcome these challenges will be the following.

In Section 2, we introduce the basics of sub-Riemannian manifolds and subLaplacians. We overcome the challenges of (i) and (ii) by introducing a unique way of choosing $L$ relative to a complemental subbundle of $\mathcal{H}$ rather than a volume form. This will have exactly the desired "lifting property". We discuss the diffusions of such operators in terms of stochastic development. In Section 3 we introduce a preferable choice of complement, which we call metric-preserving complements. Roughly speaking, such complements correspond to Riemannian foliations. While such a complement may not always exist, all sub-Riemannian manifolds discussed so far have such a complement. We give geometric conditions for when a sub-Riemannian manifold with a metric-preserving complement satisfies a generalization of the curvature-dimension inequality (1.5). From this inequality, we immediately get a result on the spectral gap of $L$ found in Section 4 . In the same section, we also apply our results to some examples.

In Part II we will look at further consequences of the curvature-dimension inequality in Theorem 3.5. A short summary of these results are given Section 5

In parallel with the development of our paper, a generalized curvature-dimension appeared in 9 ] for the case of sub-Riemannian manifolds obtained from Riemannian 
foliations with totally geodesic leaves that are of Yang-Mills type. See Remark 3.7 for details.

1.1. Notations and conventions. Unless otherwise stated, all manifolds are connected. If $\mathcal{E} \rightarrow M$ is a vector bundle over a manifold $M$, its space of smooth sections is written $\Gamma(\mathcal{E})$. If $s \in \Gamma(\mathcal{E})$ is a section, we generally prefer to write $\left.s\right|_{x}$ rather than $s(x)$ for its value in $x \in M$. By a metric tensor $\mathbf{s}$ on $\mathcal{E}$, we mean a smooth section of $\operatorname{Sym}^{2} \mathcal{E}^{*}$ which is positive definite or at least positive semi-definite. For every such metric tensor, we write $\|e\|_{\mathbf{s}}=\sqrt{\mathbf{s}(e, e)}$ for any $e \in \mathcal{E}$ even if $\mathbf{s}$ is only positive semi-definite. All metric tensors are denoted by bold, lower case Latin letters (e.g. $\mathbf{h}, \mathbf{g}, \ldots$ ). We will only use the term Riemannian metric for a positive definite metric tensor on the tangent bundle. If $\mathbf{g}$ is a Riemannian metric, we will use $\mathbf{g}^{*}, \wedge^{k} \mathbf{g}^{*}, \ldots$ for the metric tensors induced on $T^{*} M, \wedge^{k} T^{*} M, \ldots$

If $\alpha$ is a form on a manifold $M$, its contraction or interior product by a vector field $A$ will be denoted by either $\iota_{A} \alpha$ or $\alpha(A,$.$) . We use \mathcal{L}_{A}$ for the Lie derivative with respect to $A$. If $M$ is furnished with a Riemannian metric $\mathbf{g}$, any bilinear tensor $\mathbf{s}: T M \otimes T M \rightarrow \mathbb{R}$ can be identified with an endomorphism of $T M$ using $\mathbf{g}$. We use the notation $\operatorname{tr} \mathbf{s}(\times, \times)$ for the trace of this corresponding endomorphism, with the metric being implicit. If $\mathcal{H}$ is a subbundle of $T M$, we will also use the notation $\operatorname{tr}_{\mathcal{H}} \mathbf{s}(\times, \times):=\operatorname{tr} \mathbf{s}\left(\operatorname{pr}_{\mathcal{H}} \times, \operatorname{pr}_{\mathcal{H}} \times\right)$, where $\operatorname{pr}_{\mathcal{H}}$ is the orthogonal projection to $\mathcal{H}$.

\section{Sub-Riemannian manifolds AND SUb-Laplacians}

2.1. Definition of a sub-Riemannian manifolds. A sub-Riemannian manifold can be considered as a triple $(M, \mathcal{H}, \mathbf{h})$ where $M$ is a connected manifold, $\mathcal{H}$ is a subbundle of $T M$ and $\mathbf{h}$ is a positive definite metric tensor defined only on the subbundle $\mathcal{H}$. The pair $(\mathcal{H}, \mathbf{h})$ is called a sub-Riemannian structure on $M$. Any sub-Riemannian structure induces a vector bundle morphism

$$
\sharp^{\mathbf{h}^{*}}: T^{*} M \rightarrow T M,
$$

determined by the properties $\sharp \mathbf{h}^{*}\left(T^{*} M\right)=\mathcal{H}$ and $p(v)=\mathbf{h}\left(v, \sharp^{*} p\right)$ for any $p \in$ $T^{*} M$ and $v \in \mathcal{H}$. The kernel of $\sharp^{*}$ is the subbundle $\operatorname{Ann}(\mathcal{H}) \subseteq T^{*} M$ of all elements of $T^{*} M$ that vanish on $\mathcal{H}$. We can define a co-metric $\mathbf{h}^{*}$ on $T^{*} M$ by

$$
\mathbf{h}^{*}\left(p_{1}, p_{2}\right)=p_{1}\left(\sharp^{*} p_{2}\right), \quad p_{1}, p_{2} \in T_{x}^{*} M, x \in M,
$$

which obviously degenerates along $\operatorname{Ann}(\mathcal{H})$. A sub-Riemannian manifold can therefore equivalently be considered as a pair $\left(M, \mathbf{h}^{*}\right)$ where $M$ is a connected manifold and $\mathbf{h}^{*}$ a co-metric degenerating along a subbundle of $T^{*} M$. We will use both of these point of views throughout our paper, referring to the sub-Riemannian structure $(\mathcal{H}, \mathbf{h})$ and $\mathbf{h}^{*}$ interchangeably.

We will call any absolutely continuous curve $\gamma$ in $M$ horizontal if $\dot{\gamma}(t) \in \mathcal{H}_{\gamma(t)}$ for almost all $t$. We define the Carnot-Carathéodory distance $\mathrm{d}_{c c}$ on $M$ as

$$
\mathrm{d}_{c c}(x, y)=\inf _{\gamma}\left\{\int_{0}^{1} \mathbf{h}(\dot{\gamma}, \dot{\gamma})^{1 / 2} d t: \gamma(0)=x, \gamma(1)=y, \gamma \text { horizontal }\right\} .
$$

This distance is finite for any pair of points if they can be connected by at least one horizontal curve. A sufficient condition for the latter to hold is that $\mathcal{H}$ is bracketgenerating [12, 21. A subbundle $\mathcal{H}$ is called bracket-generating if its sections and their iterated brackets span the entire tangent bundle. The same property also 
guarantees that the metric topology induced by $\mathrm{d}_{c c}$ coincides with the manifold topology on $M$, however, the Hausdorff dimension of $\mathrm{d}_{c c}$ will in general be greater than the topological dimension (see e.g. [20, Th. 2.3, Th. 2.17 ]).

From now on, the rank of $\mathcal{H}$ is $n \geq 2$ while the manifold $M$ is assumed to have dimension $n+\nu$. We will refer to $\mathcal{H}$ as the horizontal bundle and its vectors and sections as horizontal and we refer to both $(\mathcal{H}, \mathbf{h})$ and $\mathbf{h}^{*}$ as a sub-Riemannian structure on $M$.

2.2. Second order operators associated to $\mathbf{h}^{*}$. For any manifold $M$, let $T^{2} M$ denote the second order tangent bundle. Sections $L \in \Gamma\left(T^{2} M\right)$ of this bundle can locally be expressed as

$$
L=\sum_{i, j=1}^{n+\nu} L_{i j} \frac{\partial}{\partial x_{i} \partial x_{j}}+\sum_{j=1}^{n+\nu} L_{j} \frac{\partial}{\partial x_{j}},
$$

relative to some local coordinate system $\left(x_{1}, \ldots, x_{n+\nu}\right)$ and functions $L_{i j}=L_{j i}$ and $L_{j}$. We can consider $T M$ as a subbundle of $T^{2} M$. Denote its inclusion by inc. This gives us a short exact sequence

$$
0 \rightarrow T M \stackrel{\text { inc }}{\longrightarrow} T^{2} M \stackrel{\text { q }}{\longrightarrow} \mathrm{Sym}^{2} T M \rightarrow 0,
$$

where $\mathbf{q}(L)=: \mathbf{q}_{L}$ is the symmetric bilinear tensor on $T^{*} M$ defined by the property

$$
\mathbf{q}_{L}(d f, d g)=\frac{1}{2}(L(f g)-f L g-g L f), \quad \text { for any } f, g \in C^{\infty}(M) .
$$

In local coordinates, we can write $\mathbf{q}_{L}(d f, d g)=\sum_{i, j=1}^{n+\nu} L_{i j} \frac{\partial f}{\partial x_{i}} \frac{\partial g}{\partial x_{j}}$ relative to the representation of $L$ in (2.1).

Let $\mathbf{h}^{*}$ be the co-metric corresponding to a sub-Riemannian structure $(\mathcal{H}, \mathbf{h})$. Then any operator $L$ satisfying $\mathbf{q}_{L}=\mathbf{h}^{*}$ can locally be written as

$$
L=\sum_{i=1}^{n} A_{i}^{2}+A_{0}
$$

where $A_{0}$ is a vector field and $A_{1}, \ldots, A_{n}$ is a local orthonormal basis of $\mathcal{H}$. From Hörmander's celebrated result [15], we know that any such operator is hypoelliptic when $\mathcal{H}$ is bracket-generating. We consider two examples where a choice of extra structure on $(M, \mathcal{H}, \mathbf{h})$ gives a differential operator of this type.

Let vol be a volume form on $M$. We define the sub-Laplacian relative to vol as the operator $\Delta_{\mathbf{h}}$ given by

$$
\Delta_{\mathbf{h}} f:=\operatorname{div} \sharp^{\mathbf{h}^{*}} d f,
$$

where $\operatorname{div} A$ is defined by $\mathcal{L}_{A} \operatorname{vol}=(\operatorname{div} A)$ vol. Any such operator satisfies

$$
\int_{M} g \Delta_{\mathbf{h}} f d \mathrm{vol}=\int_{M} f \Delta_{\mathbf{h}} g d \mathrm{vol}
$$

for any pair of functions $f, g \in C_{c}^{\infty}(M)$ of compact support. Since $L$ is also hypoelliptic, it has a smooth, symmetric heat kernel with respect to vol. This is the most common way of defining the sub-Laplacian.

We like to introduce an alternative notion of sub-Laplacian. Rather than choosing a volume, we will choose a complement $\mathcal{V}$ to $\mathcal{H}$, i.e. a subbundle $\mathcal{V}$ such that $T M=\mathcal{H} \oplus \mathcal{V}$. This choice of complement gives us projections $\operatorname{pr}_{\mathcal{H}}$ and $\operatorname{pr}_{\mathcal{V}}$ to respectively $\mathcal{H}$ and $\mathcal{V}$. A Riemannian metric $\mathbf{g}$ on $M$ is said to tame $\mathbf{h}$ if $\left.\mathbf{g}\right|_{\mathcal{H}}=\mathbf{h}$. 
Consider any Riemannian metric $\mathbf{g}$ that tames $\mathbf{h}$ and makes $\mathcal{V}$ the orthogonal complement of $\mathcal{H}$. Let $\nabla$ be the Levi-Civita connection of $\mathbf{g}$. It is simple to verify that for any pair of horizontal vector fields $A, Z \in \Gamma(\mathcal{H}), \operatorname{pr}_{\mathcal{H}} \nabla_{A} Z$ is independent of $\left.\mathbf{g}\right|_{\mathcal{V}}$. This fact allows us to define a second order operator $\Delta_{\mathbf{h}}^{\prime}$ which we call the sub-Laplacian with respect to $\mathcal{V}$. There are several ways to introduce this operator. We have chosen to define it by using a connection $\nabla^{\circ}$ which will be helpful for us later. Corresponding to the Riemannian metric $\mathbf{g}$ and the orthogonal splitting $T M=\mathcal{H} \oplus_{\perp} \mathcal{V}$, we introduce the connection

$$
\begin{aligned}
\stackrel{\circ}{\nabla}_{A} Z:= & \operatorname{pr}_{\mathcal{H}} \nabla_{\operatorname{pr}_{\mathcal{H}} A} \operatorname{pr}_{\mathcal{H}} Z+\operatorname{pr}_{\mathcal{V}} \nabla_{\operatorname{pr}_{\mathcal{V}} A} \operatorname{pr}_{\mathcal{V}} Z \\
& +\operatorname{pr}_{\mathcal{H}}\left[\operatorname{pr}_{\mathcal{V}} A, \operatorname{pr}_{\mathcal{H}} Z\right]+\operatorname{pr}_{\mathcal{V}}\left[\operatorname{pr}_{\mathcal{H}} A, \operatorname{pr}_{\mathcal{V}} Z\right] .
\end{aligned}
$$

Definition 2.1. Let $\mathcal{V}$ be a complement of $\mathcal{H}$ corresponding to the projection $\mathrm{pr}_{\mathcal{H}}$, i.e. $\mathcal{V}=\operatorname{ker}_{\mathrm{pr}_{\mathcal{H}}}$. Then the sub-Laplacian with respect to $\mathcal{V}$ is the operator

$$
\Delta_{\mathbf{h}}^{\prime} f:=\operatorname{tr}_{\mathcal{H}} \stackrel{\circ}{\nabla}_{\times, \times}^{2} f, \quad f \in C^{\infty}(M),
$$

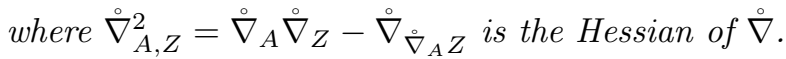

We remark that the definition only depends on the value of $\stackrel{\circ}{\nabla}_{A} Z$ when both $A$ and $Z$ take values in $\mathcal{H}$. This is illustrated by the fact that locally

$$
\Delta_{\mathbf{h}}^{\prime} f=\sum_{i=1}^{n} A_{i}^{2} f+\sum_{i, j=1}^{n} \mathbf{h}\left(\operatorname{pr}_{\mathcal{H}} \nabla_{A_{i}} A_{j}, A_{i}\right) A_{j} f
$$

where $A_{1}, \ldots, A_{n}$ is a local orthonormal basis of $\mathcal{H}$. The operator $\Delta_{\mathbf{h}}^{\prime}$ is hypoelliptic and will have a smooth heat kernel with respect to any volume form. Two different choices of complement may have the same sub-Laplacian, see Section 4.5.

In what follows, whenever we have a chosen complement $\mathcal{V}$, we will refer to it as the vertical bundle and its vectors and vector fields as vertical.

Remark 2.2. The horizontal bundle $\mathcal{H}$ is called equiregular if there exist a flag of subbundles

such that

$$
\mathcal{H}=\mathcal{H}^{1} \subseteq \mathcal{H}^{2} \subseteq \mathcal{H}^{3} \subseteq \cdots
$$

$$
\mathcal{H}^{k+1}=\operatorname{span}\left\{\left.Z\right|_{x},\left.[A, Z]\right|_{x}: Z \in \Gamma\left(\mathcal{H}^{k}\right), A \in \Gamma(\mathcal{H}), x \in M\right\} .
$$

Even if $\mathcal{H}$ is bracket-generating, it is not necessarily equiregular. We emphasize that each $\mathcal{H}^{k}$ is required to be a subbundle, and so must have constant rank. The smallest integer $r$ such that $\mathcal{H}^{r}=T M$ is called the step of $\mathcal{H}$. If $(\mathcal{H}, \mathbf{h})$ is a subRiemannian structure on $M$ with $\mathcal{H}$ equiregular, then there exist a canonical choice of volume form on $M$ called Popp's measure. For construction, see Section 4.2 or see [2] for a more detailed presentation.

2.3. Lifting property of the sub-Laplacian defined relative to a complement. Let $\pi: M \rightarrow B$ be a surjective submersion between connected manifolds $M$ and $B$. The vertical bundle of $\pi$ is the subbundle $\mathcal{V}:=\operatorname{ker} \pi_{*}$ of $T M$. An Ehresmann connection on $\pi$ is a splitting $h$ of the short exact sequence

$$
0 \longrightarrow \mathcal{V}=\operatorname{ker} \pi_{*} \longrightarrow T M \stackrel{h}{\pi_{*}} \pi^{*} T B \longrightarrow 0 .
$$

This map $h$ is uniquely determined by $\mathcal{H}=$ image $h$, which is a subbundle of $T M$ satisfying $T M=\mathcal{H} \oplus \mathcal{V}$. Hence, we refer to such a subbundle $\mathcal{H}$ as an Ehresmann 
connection as well. The image of an element $(x, \check{v}) \in \pi^{*} T B$ under $h$ is called the horizontal lift of $\check{v}$ to $x$, and denoted $h_{x} \check{v}$. Similarly, for any vector field $\check{A}$ on $B$, we have a vector field $h \check{A}$ on $M$ defined by $\left.x \mapsto h_{x} \check{A}\right|_{\pi(x)}$.

We can extend the notion of horizontal lifts to second order vectors and differential operators. If $B$ and $M$ are two manifolds, then a linear map $\varphi: T_{b}^{2} B \rightarrow T_{x}^{2} M$ is called a Schwartz morphism if $\varphi\left(T_{b} M\right) \subseteq T_{x} M$ and the following diagram commutes

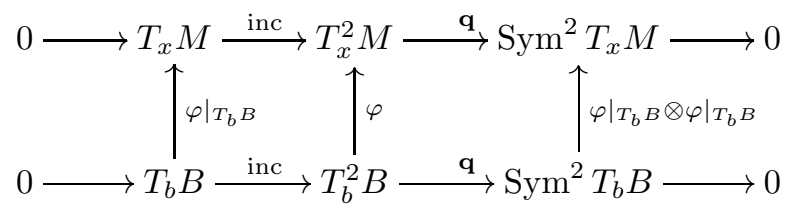

with $\mathbf{q}$ defined as in (2.2). We remark that any linear map $\varphi: T_{b}^{2} B \rightarrow T_{x}^{2} M$ is a Schwartz morphism if and only if $\varphi=\left.f_{*}\right|_{T_{b}^{2} B}$ for some map $f: B \rightarrow M$ with $f(b)=x$ (see e.g. [13, p. 80]). Let $\pi: M \rightarrow B$ be a surjective submersion. A 2 -connection on $\pi$ is then a splitting $h^{S}$ of the short exact sequence

$$
0 \longrightarrow \operatorname{ker} \pi_{*} \longrightarrow T^{2} M \stackrel{h^{S}}{\pi_{*}} \pi^{*} T^{2} B \longrightarrow 0
$$

such that $h^{S}$ is a Schwartz morphism at any point.

For any choice of Ehresmann connection $h$ on $\pi$, we can construct a corresponding 2-connection $h^{S}$ uniquely determined by the following two requirements (see e.g. [19, pp 82-83]).

- $\left.h^{S}\right|_{T M}=h$,

- $h^{S}\{\check{A}, \check{Z}\}=\{h \check{A}, h \check{Z}\}$ where $\{\check{A}, \check{Z}\}=\frac{1}{2}(\check{A} \check{Z}+\check{Z} \check{A})$ is the skew-commutator and $\check{A}, \check{Z} \in \Gamma(T B)$. Equivalently, $h^{S}(\check{A} \check{Z})=h \check{A} h \check{Z}-\frac{1}{2} \operatorname{pr}_{\mathcal{V}}[h \check{A}, h \check{Z}]$.

Using this 2-connection, we can define horizontal lifts of second order operators on $B$. We then have the following way to interpret the sub-Laplacian with respect to a complement.

Proposition 2.3. Let $\breve{\mathbf{g}}$ be a Riemannian metric on $B$ with Laplacian $\breve{\Delta}$. Relative to an Ehresmann connection $\mathcal{H}$ on $\pi$, define a sub-Riemannian structure $(\mathcal{H}, \mathbf{h})$ by $\mathbf{h}=\left.\pi^{*} \mathbf{g}\right|_{\mathcal{H}}$. Then $h^{S} \check{\Delta}=\Delta_{\mathbf{h}}^{\prime}$ where $\Delta_{\mathbf{h}}^{\prime}$ is the sub-Laplacian of $\mathcal{V}=\operatorname{ker} \pi_{*}$.

In particular, for any $f \in C^{\infty}(B)$, we have $\Delta_{\mathbf{h}}^{\prime}(f \circ \pi)=(\check{\Delta} f) \circ \pi$. A submersion $\pi:(M, \mathbf{g}) \rightarrow(B, \breve{\mathbf{g}})$ between two Riemannian manifolds such that

$$
\left.\mathbf{g}\right|_{\mathcal{H}}=\left.\pi^{*} \check{\mathbf{g}}\right|_{\mathcal{H}}, \quad \mathcal{H}=\left(\operatorname{ker} \pi_{*}\right)^{\perp}
$$

is called a Riemannian submersion. The sub-Riemannian manifolds of Proposition 2.3 can hence be considered as the result of restricting the metric on the top space in a Riemannian submersion to its horizontal subbundle.

of Proposition 2.3. Let $\mathbf{g}$ be a Riemannian metric on $M$ satisfying $\left.\mathbf{g}\right|_{\mathcal{H}}=\mathbf{h}$ and $\mathcal{H}^{\perp}=\mathcal{V}$. Let $\check{A}_{1}, \ldots, \check{A}_{n}$ be any local orthonormal basis of $T B$. Then the Laplacian can be written as

$$
\check{\Delta}=\sum_{i=1}^{n} \check{A}_{i}^{2}+\sum_{i, j=1}^{n} \check{\mathbf{g}}\left(\check{\nabla}_{\check{A}_{i}} \check{A}_{j}, \check{A}_{i}\right) \check{A}_{j}
$$


where $\check{\nabla}$ is the Levi-Civita connection of $\check{\mathbf{g}}$. However, since $\mathbf{g}\left(h \check{A}_{i}, h \check{A}_{j}\right)=\check{\mathbf{g}}\left(\check{A}_{i}, \check{A}_{j}\right)$ and since $\operatorname{pr}_{\mathcal{H}}\left[h \check{A}_{i}, h \check{A}_{j}\right]=h\left[\check{A}_{i}, \check{A}_{j}\right]$, we obtain

$$
\check{\mathbf{g}}\left(\check{\nabla}_{\check{A}_{i}} \check{A}_{j}, \check{A}_{i}\right)=\mathbf{g}\left(\stackrel{\circ}{\nabla}_{h \check{A}_{i}} h \check{A}_{j}, h \check{A}_{i}\right), \quad i, j=1,2, \ldots, n .
$$

The result follows from (2.4) and the fact that $h \check{A}_{1}, \ldots, h \check{A}_{n}$ forms a local orthonormal basis of $\mathcal{H}$.

Since the proof of Proposition 2.3 is purely local, it also holds on Riemannian foliations. To be more specific, a subbundle $\mathcal{V}$ of $T M$ is integrable if $\left[V_{1}, V_{2}\right]$ takes its values in $\mathcal{V}$ whenever $V_{1}, V_{2} \in \Gamma(\mathcal{V})$ are vertical vector fields. From the Frobenius theorem, we know that there exists a foliation $\mathcal{F}$ of $M$ consisting of immersed submanifolds of dimension $\nu=\operatorname{rank} \mathcal{V}$ such that each leaf is tangent to $\mathcal{V}$.

A Riemannian metric $\mathbf{g}$ on $M$ with a foliation induced by $\mathcal{V}$, is called bundlelike if $\mathcal{V}^{\perp}=\mathcal{H}$ and for any $A \in \Gamma(\mathcal{H})$ and $V \in \Gamma(\mathcal{V})$, we have $\left(\mathcal{L}_{V} \mathbf{g}\right)(A, A)=0$. Intuitively, one can think of a bundle-like metric $\mathbf{g}$ as a metric where $\left.\mathbf{g}\right|_{\mathcal{H}}$ does "not change" in vertical directions. A foliation $\mathcal{F}$ of a Riemannian manifold is called Riemannian if the metric is bundle-like with respect to $\mathcal{F}$. Such a manifold locally has the structure of a Riemannian submersion, that is, any point has a neighborhood $U$ such that $\pi: U \rightarrow B:=U /(\mathcal{F} \mid U)$ can be considered as a smooth submersion of manifolds. The subbundle $\left.\mathcal{H}\right|_{U}$ is an Ehresmann on $\pi$ and $B$ can be given a Riemannian metric $\check{\mathbf{g}}$ such that $\operatorname{pr}_{\mathcal{H}}^{*} \mathbf{g}=\pi^{*} \check{\mathbf{g}}$, see [22]. If we define a sub-Riemannian structure $(\mathcal{H}, \mathbf{h})$ on $M$ with $\mathbf{h}=\left.\mathbf{g}\right|_{\mathcal{H}}$, then restricted to each sufficiently small neighborhood $U$, the sub-Laplacian $\Delta_{\mathbf{h}}^{\prime}$ of $\mathcal{V}$ is equal to $h^{S} \breve{\Delta}$ were $\check{\Delta}$ is the Laplacian on $U /(\mathcal{F} \mid U)$.

Remark 2.4. By modifying the proof of Proposition 2.3 slightly, we can also get the following stronger statement: Let $\left(B, \mathcal{H}_{1}, \mathbf{h}_{1}\right)$ be a sub-Riemannian manifold and let $\pi: M \rightarrow B$ be a submersion with an Ehresmann connection $\mathcal{E}$. Define a subbundle $\mathcal{H}_{2}$ on $M$ as the horizontal lifts of all vectors in $\mathcal{H}_{1}$ with respect to $\mathcal{E}$ and let $\mathbf{h}_{2}=\left.\pi^{*} \mathbf{h}_{1}\right|_{\mathcal{H}_{2}}$ be the lifted metric. Let $\mathcal{V}_{1}$ be a choice of complement of $\mathcal{H}_{1}$ and define $\mathcal{V}_{2}$ as the direct sum of the horizontal lift of $\mathcal{V}_{1}$ and ker $\pi_{*}$. Then, if $\Delta_{\mathcal{H}_{j}}^{\prime}$ is the sub-Laplacian with respect to $\mathcal{V}_{j}, j=1,2$, we have $\Delta_{\mathcal{H}_{2}}^{\prime}=h^{S} \Delta_{\mathcal{H}_{1}}^{\prime}$, where $h^{S}$ is also defined with respect to $\mathcal{E}$.

2.4. Comparison between the sub-Laplacian of a complement and a volume form. Let $(M, \mathcal{H}, \mathbf{h})$ be a sub-Riemannian manifold and let $\mathbf{g}$ be a Riemannian metric taming $\mathbf{h}$. Let $\Delta_{\mathbf{h}}$ be the sub-Laplacian defined with respect to the volume form of $\mathbf{g}$ and let $\Delta_{\mathbf{h}}^{\prime}$ be defined relative to the complement $\mathcal{H}^{\perp}=\mathcal{V}$. We introduce a vector field $N$ by formula

$$
\Delta_{\mathbf{h}}=\Delta_{\mathbf{h}}^{\prime}-N .
$$

It can then be verified that $N$ is horizontal and can be defined by the relation

$$
\mathbf{g}(A, N)=-\frac{1}{2} \operatorname{tr} \mathcal{V}\left(\mathcal{L}_{\mathrm{pr}_{\mathcal{H}} A} \mathbf{g}\right)(\times, \times) .
$$

In order for $\Delta_{\mathbf{h}}^{\prime}$ to be the sub-Laplacian with respect to some volume form, we must have $N=-\sharp^{*}$ ( $d \phi$ for some function $\phi \in C^{\infty}(M)$. Indeed, if $\operatorname{div} A$ denotes the divergence of a vector field $A$ with respect to vol, then $\operatorname{div} A+d \phi(A)$ is its divergence with respect to $e^{\phi}$ vol. It follows that the sub-Laplacian of $e^{\phi}$ vol is given as $\Delta_{\mathbf{h}} f+\left(\sharp^{\mathbf{h}^{*}} d \phi\right) f$. 
Remark 2.5. Let $(M, \mathbf{g})$ be a Riemannian manifold with a foliation given by an integrable subbundle $\mathcal{V}$. Assume that $\mathbf{g}$ is bundle-like relative to $\mathcal{V}$. Let $\mathcal{H}$ be the orthogonal complement of $\mathcal{V}$ and define $\mathbf{h}=\left.\mathbf{g}\right|_{\mathcal{H}}$. Write vol for the volume form of $\mathbf{g}$. Let $\Delta_{\mathbf{h}}$ and $\Delta_{\mathbf{h}}^{\prime}$ be the sub-Laplacian of $(M, \mathcal{H}, \mathbf{h})$ relative to respectively vol and $\mathcal{V}$. Then $N=\Delta_{\mathrm{h}}^{\prime}-\Delta_{\mathrm{h}}$ is the mean curvature vector field of the leaves of the foliations by (2.5). Hence, the operators $\Delta_{\mathbf{h}}$ and $\Delta_{\mathbf{h}}^{\prime}$ coincide in this case if and only if the leafs of the foliation are minimal submanifolds.

2.5. Diffusion of $\Delta_{\mathbf{h}}^{\prime}$. Let $L$ be any section of $T^{2} M$ with $\mathbf{q}_{L}$ being positive semidefinite and let $x \in M$ be any point. Then, by [16, Theorems 1.3.4 and 1.3.6] there exists an $L$-diffusion $X_{t}=X_{t}(x)$ satisfying $X_{0}=x$, unique in law, defined up to some explosion time $\tau=\tau(x)$. An L-diffusion $X_{t}$ is an $M$-valued semimartingale up to some stopping time $\tau$ defined on some filtered probability space $(\Omega, \mathscr{F}, \mathbb{P})$, such that for any $f \in C^{\infty}(M)$,

$$
M_{t}^{f}:=f\left(X_{t}\right)-f\left(X_{0}\right)-\int_{0}^{t} L f\left(X_{s}\right) d s, \quad 0 \leq t<\tau,
$$

is a local martingale up to $\tau$. We will always assume that $\tau$ is maximal, so that $\tau$ is the explosion time, i.e. $\{\tau<\infty\} \subseteq\left\{\lim _{t \uparrow \tau} X_{t}=\infty\right\}$ almost surely.

Let $\Delta_{\mathbf{h}}^{\prime}$ be the sub-Laplacian defined with respect to a choice of complement $\mathcal{V}$. Let $\mathbf{g}$ be a Riemannian metric such that $\mathcal{H}^{\perp}=\mathcal{V}$ and $\left.\mathbf{g}\right|_{\mathcal{H}}=\mathbf{h}$. Define $\stackrel{\circ}{\nabla}^{\circ}$ on as in (2.3). To simplify our presentation, we will assume that $\nabla^{\circ} \mathbf{g}=0$. See Remark 2.6 for the general case. For a given point $x \in M$, let $\gamma(t)$ be any smooth curve in $M$ with $\gamma(0)=x$. Let $\phi_{1}, \ldots, \phi_{n}$ and $\psi_{1}, \ldots, \psi_{\nu}$ be orthonormal bases for respectively $\left.\mathcal{H}\right|_{x}$ and $\left.\mathcal{V}\right|_{x}$. Parallel transport of such bases remain orthonormal bases from our assumption $\stackrel{\circ}{\nabla} \mathbf{g}=0$.

Define $\mathrm{O}(n) \rightarrow \mathrm{O}(\mathcal{H}) \rightarrow M$ as the bundle of orthonormal frames of $\mathcal{H}$, and define $\mathrm{O}(\nu) \rightarrow \mathrm{O}(\mathcal{V}) \rightarrow M$ similarly. Let

$$
\mathrm{O}(n) \times \mathrm{O}(\nu) \rightarrow \mathrm{O}(\mathcal{H}) \odot \mathrm{O}(\mathcal{V}) \stackrel{\pi}{\rightarrow} M
$$

denote the product bundle. We can then define an Ehresmann connection $\mathcal{E}^{\circ}$ on $\pi$ such that a curve $(\phi(t), \psi(t))=\left(\phi_{1}(t), \ldots, \phi_{n}(t), \psi_{1}(t), \ldots, \psi_{\nu}(t)\right)$ in $\mathrm{O}(\mathcal{H}) \odot \mathrm{O}(\mathcal{V})$ is tangent to $\mathcal{E}^{\circ}$ if and only if each $\phi_{j}(t), 1 \leq j \leq n$, and $\psi_{s}(t), 1 \leq s \leq \nu$, is parallel along $\gamma(t)=\pi(\phi(t), \psi(t))$.

Define vector fields $\widetilde{A}_{1}, \ldots, \widetilde{A}_{n}$ on $\mathrm{O}(\mathcal{H}) \odot \mathrm{O}(\mathcal{V})$ by $\left.\widetilde{A}_{j}\right|_{\phi, \psi}=h_{\phi, \psi} \phi_{j}$ where the horizontal lift is with respect to $\mathcal{E}^{\triangleright}$. For any $x \in M$ and $\left.(\phi, \psi) \in \mathrm{O}(\mathcal{H}) \odot \mathrm{O}(\mathcal{V})\right|_{x}$, consider the solution $\Phi_{t}$ of the Stratonovich SDE up to explosion time $\tau$,

$$
d \Phi_{t}=\left.\sum_{j=1}^{n} \widetilde{A}_{j}\right|_{\Phi_{t}} \circ d W_{t}^{j}, \quad \Phi_{0}=(\phi, \psi),
$$

where $W=\left(W^{1}, \ldots, W^{n}\right)$ is a Brownian motion in $\mathbb{R}^{n}$ with $W_{0}=0$. It is then simple to verify that $\Phi$ is a $\frac{1}{2} h^{S} \Delta_{\mathbf{h}}^{\prime}$-diffusion since $h^{S} \Delta_{\mathbf{h}}^{\prime}=\sum_{i=1}^{n} \widetilde{A}_{j}^{2}$ if we consider the 2-connection $h^{S}$ induced by the Ehresmann connection $\mathcal{E}^{\dot{\nabla}}$. This shows that $X_{t}=\pi\left(\Phi_{t}\right)$ is an $\frac{1}{2} \Delta_{\mathbf{h}}^{\prime}$-diffusion on $M$ with $X_{0}=x$. Note that $\tau$ will be the explosion time of $X_{t}$ as well by [23].

Remark 2.6. If $\nabla^{\circ} \mathbf{g} \neq 0$, we can instead use the connection

$$
\nabla_{A}^{\infty} Z:=\operatorname{pr}_{\mathcal{H}} \nabla_{A} \operatorname{pr}_{\mathcal{H}} Z+\operatorname{pr}_{\mathcal{V}} \nabla_{A} \operatorname{pr}_{\mathcal{V}} Z, \quad A, Z \in \Gamma(T M) .
$$


It clearly satisfies $\nabla^{\infty} \mathbf{g}=0$, preserves the horizontal and vertical bundle under parallel transport and has $\stackrel{\circ}{\nabla}_{A} Z=\nabla_{A} Z$ for any pair horizontal vector fields $A, Z \in \Gamma(\mathcal{H})$. The definition of $\Delta_{\mathbf{h}}$ in Definition 2.1 hence remains the same if we replace $\nabla^{\circ}$ with $\nabla^{\circ}$. The reason we will prefer to use $\nabla$ is the property given in Lemma 3.2 (a) which fails for $\nabla^{\infty}$.

Remark 2.7. Instead of using the lift to $\mathrm{O}(\mathcal{H}) \odot \mathrm{O}(\mathcal{V})$, we could have considered the full frame bundle $\mathrm{O}(T M)$ and development with respect to $\stackrel{\circ}{\nabla}$ or $\stackrel{\infty}{\nabla}^{\circ}$, see e.g. [16. Section 2.3]. The diffusion of $\frac{1}{2} \Delta_{\mathbf{h}}^{\prime}$ then has the Brownian motion in an $n$ dimensional subspace of $\mathbb{R}^{n+\nu}$ as its anti-development, where the subspace depends on the choice of initial frame.

Remark 2.8. If $\Delta_{\mathbf{h}}^{\prime}$ is symmetric with respect to some volume form vol, the the following observation made in [10, Theorem 4.4] guarantees us that the diffusion $X_{t}$ has infinite lifetime, i.e. $\tau=\infty$ a.s. Let $\mathbf{g}$ be any Riemannian metric on $M$ taming $\mathbf{h}$ with corresponding volume form vol. Assume that $\mathbf{g}$ is complete with (Riemannian) Ricci curvature bounded from below. Note that if $\mathrm{d}_{\mathbf{g}}$ is the metric of $\mathbf{g}$ and $\mathrm{d}_{c c}$ is the Carnot-Carathéodory of $\mathbf{h}$, then $\mathrm{d}_{c c}(x, y) \geq \mathrm{d}_{\mathbf{g}}(x, y)$ for any $(x, y) \in M \times M$. Hence, $B_{r}(x) \subseteq B_{r}^{\mathbf{g}}(x)$ where $B_{r}(x)$ and $B_{r}^{\mathbf{g}}(x)$ are the balls of respectively $\mathrm{d}_{c c}$ and $\mathrm{d}_{\mathrm{g}}$, centered at $x$ with radius $r$. By the Riemannian volume comparison theorem, we have

$$
\operatorname{vol}\left(B_{r}(x)\right) \leq \operatorname{vol}\left(B_{r}^{\mathbf{g}}(x)\right) \leq C_{1} e^{C_{2} r}
$$

for some constants $C_{1}, C_{2}$. In conclusion, $\int_{0}^{\infty} \frac{r}{\log \operatorname{vol}\left(B_{r}(x)\right)} d r=\infty$ and so 25, Theorem 3] tells us that $\frac{1}{2} \Delta_{\mathbf{h}}^{\prime}$-diffusions $X_{t}$ starting at a point $x \in M$ has infinite lifetime.

\section{RiEmannian Foliations and the CURVATURE-DIMENSION INEQUALITY}

3.1. Riemannian foliations and the geometry of $\nabla^{\circ}$. Let $(M, \mathcal{H}, \mathbf{h})$ be a subRiemannian manifold with a complement $\mathcal{V}$. Let $\Delta_{\mathbf{h}}^{\prime}$ be the sub-Laplacian relative to $\mathcal{V}$. In order to introduce a curvature-dimension inequality for $\Delta_{\mathbf{h}}^{\prime}$, we will need to choose a Riemannian metric on $M$ which tame $\mathbf{h}$ and makes $\mathcal{H}$ and $\mathcal{V}$ orthogonal. Choose a metric tensor $\mathbf{v}$ on $\mathcal{V}$ to obtain a Riemannian metric $\mathbf{g}=\operatorname{pr}_{\mathcal{H}}^{*} \mathbf{h}+\operatorname{pr}_{\mathcal{V}}^{*} \mathbf{v}$.

We make the following assumptions on $\mathcal{V}$. We want to consider the specific case when $\mathcal{V}$ is integrable and satisfies

$$
\mathcal{L}_{V}\left(\operatorname{pr}_{\mathcal{H}}^{*} \mathbf{h}\right)=0 \quad \text { for any } V \in \Gamma(\mathcal{V})
$$

Then $\mathbf{g}=\operatorname{pr}_{\mathcal{H}}^{*} \mathbf{h}+\operatorname{pr}_{\mathcal{V}}^{*} \mathbf{v}$ is bundle-like for any choice of $\mathbf{v}$, giving us a Riemannian foliation as defined in Section 2.3. Since this property is independent of $\mathbf{g} \mid \mathcal{V}$, we introduce the following definition.

Definition 3.1. An integrable subbundle $\mathcal{V}$ is called a metric-preserving complement to the sub-Riemannian manifold $(M, \mathcal{H}, \mathbf{h})$ if $T M=\mathcal{H} \oplus \mathcal{V}$ and (3.1) hold.

In the special case when the foliation $\mathcal{F}$ of $\mathcal{V}$ gives us a submersion $\pi: M \rightarrow$ $B=M / \mathcal{F}$ with $\mathcal{H}$ as an Ehresmann connection on $\pi$, the curvature of $\mathcal{H}$ is a vector-valued two-form $\mathcal{R} \in \Gamma\left(\bigwedge^{2} T^{*} M \otimes T M\right)$ defined by

$$
\mathcal{R}(A, Z):=\operatorname{pr}_{\mathcal{V}}\left[\operatorname{pr}_{\mathcal{H}} A, \operatorname{pr}_{\mathcal{H}} Z\right], \quad A, Z \in \Gamma(T M) .
$$


This curvature measures how far $\mathcal{H}$ is from being a flat connection, i.e. an integrable subbundle. We will call $\mathcal{R}$ given by formula (3.2) the curvature of $\mathcal{H}$ even when $\mathcal{V}$ does not give us a submersion globally.

Define $\stackrel{\circ}{\nabla}$ relative to $\mathrm{g}$ as in (2.3). The following properties are simple to verify.

Lemma 3.2. Let $\mathbf{g}$ be a Riemannian metric and let $\mathcal{V}$ be an integrable subbundle of $T M$ with orthogonal complement $\mathcal{H}$. Define $\stackrel{\circ}{\nabla}$ relative to $\mathbf{g}$ and the splitting $T M=\mathcal{H} \oplus \mathcal{V}$. Write $\mathbf{h}$ and $\mathbf{v}$ for the restriction of $\mathbf{g}$ to respectively $\mathcal{H}$ and $\mathcal{V}$.

(a) Let $Z \in \Gamma(T M)$ be an arbitrary vector field. If $A \in \Gamma(\mathcal{H})$ is horizontal, both $\stackrel{\circ}{ }_{A} Z$ and $\stackrel{\circ}{Z}_{Z} A$ only depends on $\mathbf{h}$ and the splitting $T M=\mathcal{H} \oplus \mathcal{V}$. They are independent of $\mathbf{v}$. Similarly, if $V$ is vertical, then $\stackrel{\circ}{\nabla}_{V} Z$ and $\nabla_{Z} V$ are independent of $\mathbf{h}$.

(b) The torsion of $\stackrel{\circ}{\nabla}$ is given as $T^{\circ}(A, Z)=-\mathcal{R}(A, Z)$.

(c) $\mathcal{V}$ is a metric-preserving complement of $(M, \mathcal{H}, \mathbf{h})$ if and only if

$$
\left(\stackrel{\circ}{\nabla}_{A} \mathbf{g}\right)(Z, Z)=\left(\mathcal{L}_{\mathrm{pr}_{\mathcal{H}} A} \mathbf{g}\right)\left(\operatorname{pr}_{\mathcal{V}} Z, \operatorname{pr}_{\mathcal{V}} Z\right) .
$$

Equivalently, $\mathcal{V}$ is metric-preserving if and only if $\stackrel{\circ}{\nabla}^{*}=0$.

(d) If $\mathcal{V}$ is metric-preserving, then

$$
\left(\stackrel{\circ}{\nabla}_{Z_{1}} \mathbf{g}\right)\left(Z_{2}, Z_{3}\right)=-2 \mathbf{g}\left(Z_{1}, \mathrm{II}\left(\operatorname{pr}_{\mathcal{V}} Z_{2}, \operatorname{pr}_{\mathcal{V}} Z_{3}\right)\right)
$$

where II is the second fundamental form of the foliation of $\mathcal{V}$.

Recall that when $\mathcal{V}$ is metric-preserving, g is bundle-like. We write down the basic properties of the curvature $R^{\circ}$ of $\stackrel{\circ}{\nabla}^{\circ}$ when $\mathcal{V}$ is metric-preserving.

Lemma 3.3. Let $Z_{1}, Z_{2} \in \Gamma(T M)$ be arbitrary vector fields and let $A \in \Gamma(\mathcal{H})$ be a horizontal vector field. Then

(a) $\mathbf{g}\left(R^{\stackrel{\circ}{\nabla}}\left(Z_{1}, Z_{2}\right) A, A\right)=0$,

(b) $\mathbf{g}\left(R^{\circ}\left(A, Z_{1}\right) Z_{2}, A\right)-\mathbf{g}\left(R^{\circ}\left(A, Z_{2}\right) Z_{1}, A\right)=0$.

In particular, $\mathbf{g}\left(R^{\circ}\left(A, \operatorname{pr}_{\mathcal{V}} Z_{2}\right) Z_{1}, A\right)=0$.

Proof. The statement in (a) holds since $\left(\nabla^{\circ} \mathbf{g}\right)\left(\operatorname{pr}_{\mathcal{H}} \cdot, \mathrm{pr}_{\mathcal{H}} \cdot\right)=0$. For the identity in (b), we recall the first Bianchi identity for connections with torsion,

$$
\circlearrowright R^{\stackrel{\circ}{\nabla}}\left(A, Z_{1}\right) Z_{2}=-\circlearrowright T^{\stackrel{\circ}{\nabla}}\left(A, T^{\stackrel{\circ}{\nabla}}\left(Z_{1}, Z_{2}\right)\right)+\circlearrowright\left(\stackrel{\circ}{\nabla}_{A} T^{\stackrel{\circ}{\nabla}}\right)\left(Z_{1}, Z_{2}\right),
$$

where $\circlearrowright$ denotes the cyclic sum. This means that

$$
\begin{aligned}
& \mathbf{g}\left(\circlearrowright R^{\stackrel{\circ}{\nabla}}\left(A, Z_{1}\right) Z_{2}, A\right)=\mathbf{g}\left(R^{\stackrel{\circ}{\nabla}}\left(A, Z_{1}\right) Z_{2}, A\right)-\mathbf{g}\left(R^{\stackrel{\circ}{\nabla}}\left(A, Z_{2}\right) Z_{1}, A\right) \\
& \quad=\mathbf{g}\left(-\circlearrowright T^{\stackrel{\circ}{\nabla}}\left(A, T^{\stackrel{\circ}{\nabla}}\left(Z_{1}, Z_{2}\right)\right)+\circlearrowright\left(\stackrel{\circ}{\nabla}_{A} T^{\stackrel{\circ}{\nabla}}\right)\left(Z_{1}, Z_{2}\right), A\right)=0 .
\end{aligned}
$$

We will use the fact that we have a clear idea of what Ricci curvature is on a Riemannian manifold, to introduce a corresponding tensor on a sub-Riemannian manifold with a metric-preserving complement $\mathcal{V}$.

Proposition 3.4. Introduce a tensor $\operatorname{Ric}_{\mathcal{H}} \in \Gamma\left(T^{*} M^{\otimes 2}\right)$ by

$$
\operatorname{Ric}_{\mathcal{H}}\left(Z_{1}, Z_{2}\right)=\operatorname{tr} R^{\stackrel{\circ}{\nabla}}\left(\operatorname{pr}_{\mathcal{H}} \cdot, Z_{2}\right) Z_{1} .
$$

Then

(a) $\operatorname{Ric}_{\mathcal{H}}$ is symmetric and $\mathcal{V} \subseteq \operatorname{ker} \operatorname{Ric}_{\mathcal{H}}$. 
(b) $\operatorname{Ric}_{\mathcal{H}}$ is independent of choice of metric $\mathbf{v}$ on $\mathcal{V}$.

(c) Let $\mathcal{F}$ be the foliation induced by $\mathcal{V}$. Let $U$ be any neighborhood of $M$ such that the quotient map $\pi: U \rightarrow B:=U /(\mathcal{F} \mid U)$ is a smooth submersions of manifolds. Let $\widetilde{\text { Ric }}$ be the Ricci curvature on $B$ with respect to the induced Riemannian structure. Then $\left.\operatorname{Ric}_{\mathcal{H}}\right|_{U}=\pi^{*}$ Ric.

Proof. Since $\nabla^{\circ}$ preserves both the vertical and horizontal bundle, and by means of Lemma 3.3 (b), we know that $\operatorname{Ric}_{\mathcal{H}}=\operatorname{pr}_{\mathcal{H}}^{*} \operatorname{Ric}_{\mathcal{H}}$. It is symmetric by Lemma 3.3 (b), which completes the proof of the statement in (a). The statement (b) can be verified using the definition of the Levi-Civita connection.

To prove (c), let $\check{A}_{1}, \ldots, \check{A}_{n}$ be any local orthonormal basis on $B$. Note that

$$
\left[h \check{A}_{i}, h \check{A}_{j}\right]=h\left[\check{A}_{i}, \check{A}_{j}\right]+\mathcal{R}\left(h \check{A}_{i}, h \check{A}_{j}\right) .
$$

Also, for any $V \in \Gamma(\mathcal{V}), \stackrel{\circ}{\nabla}_{V} h \check{A}_{i}=\operatorname{pr}_{\mathcal{H}}\left[V, h \check{A}_{i}\right]=0$ since $h \check{A}_{i}$ and $V$ are $\pi$-related to respectively $\check{A}_{i}$ and the zero-section of $T B$. Finally, recall that $\stackrel{\circ}{\nabla}_{h A_{i}} h A_{j}=h \breve{\nabla}_{A_{i}} A_{j}$ from the proof of Proposition 2.3. For any $j, k$,

$$
\begin{aligned}
& \sum_{i=1}^{n} \mathbf{g}\left(R^{\stackrel{\circ}{\nabla}}\left(h \check{A}_{i}, h \check{A}_{j}\right) h \check{A}_{k}, h \check{A}_{i}\right) \\
& \quad=\sum_{i=1}^{n} \mathbf{g}\left(\left[\check{\circ}_{h \check{A}_{i}}, \stackrel{\circ}{\nabla}_{h \check{A}_{j}}\right] h \check{A}_{k}-\stackrel{\circ}{\nabla}_{h\left[\check{A}_{i}, \check{A}_{j}\right]} h \check{A}_{k}-\stackrel{\circ}{\nabla}_{\mathcal{R}\left(h \check{A}_{i}, h \check{A}_{j}\right)} h \check{A}_{k}, h \check{A}_{i}\right) \\
& \quad=\sum_{i=1}^{n} \check{\mathbf{g}}\left(\left[\check{\nabla}_{\check{A}_{i}}, \check{\nabla}_{\check{A}_{j}}\right] \check{A}_{k}-\check{\nabla}_{\left[\check{A}_{i}, \check{A}_{j}\right]} \check{A}_{k}, \check{A}_{i}\right)=\widetilde{\operatorname{Ric}}\left(\check{A}_{k}, \check{A}_{j}\right) .
\end{aligned}
$$

It follows that $\operatorname{Ric}_{\mathcal{H}}\left(h \check{A}_{k}, h \check{A}_{j}\right)=\widetilde{\operatorname{Ric}}\left(\pi_{*} h \check{A}_{k}, \pi_{*} h \check{A}_{j}\right)$, and hence the same holds for any pair of vector fields $Z_{1}, Z_{2}$.

3.2. A generalized curvature-dimension inequality. For any symmetric bilinear tensor $\mathbf{s}^{*} \in \Gamma\left(\operatorname{Sym}^{2} T M\right)$, we associate a symmetric map $\Gamma^{\mathbf{s}^{*}}$ of smooth functions by

$$
\begin{array}{ccc}
\Gamma^{\mathrm{s}^{*}}: \quad C^{\infty}(M) \times C^{\infty}(M) & \rightarrow & C^{\infty}(M) \\
(f, g) & \mapsto & \mathbf{s}^{*}(d f, d g) .
\end{array}
$$

By Leibniz identity, we have relation $\Gamma^{\mathbf{s}^{*}}(f, g \phi)=g \Gamma^{\mathbf{s}^{*}}(f, \phi)+\phi \Gamma^{\mathbf{s}^{*}}(f, g)$ for arbitrary smooth functions $f, g, \phi$. Relative to some second order operator $L \in \Gamma\left(T^{2} M\right)$, we define

$$
\Gamma_{2}^{\mathbf{s}^{*}}(f, g):=\frac{1}{2}\left(L \Gamma^{\mathbf{s}^{*}}(f, g)-\Gamma^{\mathbf{s}^{*}}(L f, g)-\Gamma^{\mathbf{s}^{*}}(f, L g)\right) .
$$

To simplify notation, we will write $\Gamma^{\mathrm{s}^{*}}(f, f)=\Gamma^{\mathrm{s}^{*}}(f)$ and $\Gamma_{2}^{\mathrm{s}^{*}}(f, f)=\Gamma_{2}^{\mathrm{s}^{*}}(f)$.

Let $\mathbf{h}^{*}=\mathbf{q}_{L}$ where $\mathbf{q}$ is defined as in (2.2). Assume that $\mathbf{h}^{*}$ is positive semidefinite and let $\mathbf{v}^{*} \in \Gamma\left(\operatorname{Sym}^{2} T M\right)$ be another chosen positive semi-definite section. Then $L$ is said to satisfy a generalized curvature-dimension inequality with parameters $n, \rho_{1}, \rho_{2,0}$ and $\rho_{2,1}$ if

$$
\left(C D^{*}\right) \quad \Gamma_{2}^{\mathbf{h}^{*}}(f)+\ell \Gamma_{2}^{\mathbf{v}^{*}}(f) \geq \frac{1}{n}(L f)^{2}+\left(\rho_{1}-\frac{1}{\ell}\right) \Gamma^{\mathbf{h}^{*}}(f)+\left(\rho_{2,0}+\rho_{2,1} \ell\right) \Gamma^{\mathbf{v}^{*}}(f),
$$

for any $\ell>0$. We include the possibility of $n=\infty$. Any such inequality implies $\Gamma_{2}^{\mathbf{v}^{*}}(f) \geq \rho_{2,1} \Gamma^{\mathbf{v}^{*}}(f)$ by dividing both sides with $\ell$ and letting it go to infinity. 
Let $(M, \mathcal{H}, \mathbf{h})$ be a sub-Riemannian manifolds with an integrable complement $\mathcal{V}$ that is also metric-preserving. Choose a metric $\mathbf{v}$ on $\mathcal{V}$, and define $\stackrel{\circ}{\nabla}^{\circ}$ with respect to the corresponding Riemannian metric $\mathbf{g}$. Let $\mathbf{v}^{*}$ be the co-metric corresponding to $\mathbf{v}$. Using the properties of ${ }^{\circ}$, we are ready to present our generalized curvaturedimension inequality. We will make the following assumptions on $(M, \mathcal{H}, \mathbf{h})$.

(i) Let $\mathcal{R}$ be the curvature of $\mathcal{H}$ relative to the complement $\mathcal{V}$. Assume that the length of $\mathcal{R}$ is bounded on $M$ and define $\mathscr{M}_{\mathcal{R}}<\infty$ as the minimal number such that

$$
\|\mathcal{R}(v, \cdot)\|_{\mathbf{g}^{*} \otimes \mathbf{g}} \leq \mathscr{M}_{\mathcal{R}}\left\|\operatorname{pr}_{\mathcal{H}} v\right\|_{\mathbf{g}}, \quad \text { for any } v \in T M .
$$

By replacing $\mathbf{v}$ with $\mathscr{M}_{\mathcal{R}}^{-2} \mathbf{v}$, we may assume that $\mathscr{M}_{\mathcal{R}}=1$. From now on, we will work with the vertical metric $\mathbf{v}$ normalized in this way.

(ii) Let Ric $\mathcal{H}_{\mathcal{H}}$ be defined as in Proposition 3.4. Assume that Ric H $_{\mathcal{H}}$ has a lower bound $\rho_{\mathcal{H}}$, i.e. for every $v \in T M$, we have

$$
\operatorname{Ric}_{\mathcal{H}}(v, v) \geq \rho_{\mathcal{H}}\left\|\operatorname{pr}_{\mathcal{H}} v\right\|_{\mathbf{h}}^{2} .
$$

(iii) Assume that the length of the tensor $\nabla^{\circ} \mathbf{g}^{*}\left(=\stackrel{\nabla}{\nabla}^{*}\right)$ is bounded. Write

$$
\mathscr{M}_{\nabla}^{\circ \mathbf{v}^{*}}=\sup _{M}\left\|\stackrel{\circ}{\nabla} \cdot \mathbf{v}^{*}(\cdot, \cdot)\right\|_{\mathbf{g}^{*} \otimes \operatorname{Sym}^{2} \mathbf{g}^{*}}
$$

Define also

$$
\left(\Delta_{\mathbf{h}}^{\prime} \mathbf{v}^{*}\right)(p, p)=\operatorname{tr}_{\mathcal{H}}\left(\stackrel{\circ}{\nabla}_{\times, \times}^{2} \mathbf{v}^{*}\right)(p, p)
$$

and assume that for any $p \in T^{*} M$, we have $\left(\Delta_{\mathbf{h}}^{\prime} \mathbf{v}^{*}\right)(p, p) \geq \rho_{\Delta_{\mathbf{h}}^{\prime} \mathbf{v}^{*}}\|p\|_{\mathbf{v}^{*}}^{2}$ globally on $M$ for some constant $\rho_{\Delta_{\mathrm{h}}^{\prime}} \mathbf{v}^{*}$.

(iv) Finally, introduce $\operatorname{Ric}_{\mathcal{H} \mathcal{V}}$ as

$$
\operatorname{Ric}_{\mathcal{H} \mathcal{V}}\left(Z_{1}, Z_{2}\right)=\frac{1}{2} \operatorname{tr}\left(\mathbf{g}\left(Z_{1},\left(\stackrel{\circ}{\nabla}_{\times} \mathcal{R}\right)\left(\times, Z_{2}\right)\right)+\mathbf{g}\left(Z_{2},\left(\stackrel{\circ}{\nabla}_{\times} \mathcal{R}\right)\left(\times, Z_{1}\right)\right)\right),
$$

Assume that for any $Z \in \Gamma(T M)$,

$$
\operatorname{Ric}_{\mathcal{H V}}(Z, Z) \geq-2 \mathscr{M}_{\mathcal{H} \mathcal{V}}\left\|\operatorname{pr}_{\mathcal{V}} Z\right\|_{\mathbf{v}}\left\|\operatorname{pr}_{\mathcal{H}} Z\right\|_{\mathbf{h}}
$$

holds pointwise on $M$ for some number $\mathscr{M}_{\mathcal{H} \mathcal{V}}$.

Note that $\mathscr{M}_{\mathcal{R}}, \mathscr{M}_{\nabla_{\mathbf{v}^{*}}}$ and $\mathscr{M}_{\mathcal{H} \mathcal{V}}$ are always non-negative, while this is not necessarily true for $\rho_{\mathcal{H}}$ and $\rho_{\Delta_{\mathbf{h}}^{\prime} \mathbf{v}^{*}}$. We will define one more constant, which will always exist. For any $\alpha \in \Gamma\left(T^{*} M\right)$, define $m_{\mathcal{R}}$ as the maximal number satisfying

$$
\|\alpha(\mathcal{R}(., .))\|_{\wedge^{2} \mathbf{h}^{*}} \geq m_{\mathcal{R}}\|\alpha\|_{\mathbf{v}^{*}}
$$

If $\operatorname{rank} \mathcal{V}=\nu$, then

$$
\nu m_{\mathcal{R}}^{2} \leq\|\mathcal{R}\|_{\wedge^{2} \mathbf{g}^{*} \otimes \mathbf{g}}^{2} \leq \frac{n}{2} \mathscr{M}_{\mathcal{R}}^{2}=\frac{n}{2},
$$

so the maximal value of $m_{\mathcal{R}}$ is $\left(\frac{n}{2 \nu}\right)^{1 / 2}$ when the vertical metric has been normalized. Moreover, it can only be nonzero if $\mathcal{H}$ is step 2 equiregular as defined in Remark 2.2

With these assumptions in place, we have the following version of a generalized curvature-dimension inequality. 
Theorem 3.5. Define $\Gamma_{2}^{\mathbf{s}^{*}}$ with respect to $L=\Delta_{\mathbf{h}}^{\prime}$. Then $L$ satisfies (CD*) with

$$
\left\{\begin{aligned}
n & =\operatorname{rank} \mathcal{H} \\
\rho_{1} & =\rho_{\mathcal{H}}-c^{-1}, \\
\rho_{2,0} & =\frac{1}{2} m_{\mathcal{R}}^{2}-c\left(\mathscr{M}_{\mathcal{H} \mathcal{V}}+\mathscr{M}_{\nabla \mathbf{v}^{*}}\right)^{2}, \\
\rho_{2,1} & =\frac{1}{2} \rho_{\Delta_{\mathbf{h}}^{\prime} \mathbf{v}^{*}}-\mathscr{M}_{\dot{\nabla} \mathbf{v}^{*}}^{2},
\end{aligned}\right.
$$

for any positive $c>0$.

Note that we include the possibility $c=\infty$ when $\mathscr{M}_{\mathcal{H V}}=\mathscr{M}_{{ }_{\nabla} \mathbf{v}^{*}}=0$. The proof is found in Section 3.5 .

We can give the following interpretation of the different terms in the inequality.

(i) $\mathscr{M}_{\mathcal{R}}$ and $m_{\mathcal{R}}$ measures how well $\mathbf{v}$ can be controlled by the curvature $\mathcal{R}$ of $\mathcal{H}$. To be more precise, for any $x \in M, p \in T_{x}^{*} M$, define $C_{1}(x)$ and $C_{2}(x)$ such that

$$
C_{1}(x)\|p\|_{\mathbf{v}^{*}} \leq\|p \circ \mathcal{R}\|_{\wedge^{2} \mathbf{h}^{*}} \leq C_{2}(x)\|p\|_{\mathbf{v}^{*}}
$$

with $C_{1}(x)$ maximal and $C_{2}(x)$ minimal at every point. Then

$$
\frac{n}{2} \mathscr{M}_{\mathcal{R}} \geq \sup _{M} C_{2}(x)
$$

while $m_{\mathcal{R}}=\inf _{M} C_{1}(x)$.

(ii) $\mathrm{Ric}_{\mathcal{H}}$ is a generalization of "the Ricci curvature downstairs" on sub-Riemannian structures on submersions by Proposition 3.4 (c).

(iii) Both $\mathscr{M}_{\nabla \mathbf{v}^{*}}$ and $\rho_{\Delta_{\mathbf{h}}^{\prime} \mathbf{v}^{*}}$ measure how $\mathbf{v}$ changes in horizontal directions. In particular, $\stackrel{\circ}{\nabla} \mathbf{v}^{*}$ is the second fundamental form by Lemma 3.2 ,

(iv) $\operatorname{Ric}_{\mathcal{H V}}$ measures how "optimal" our subbundle $\mathcal{H}$ is with respect to our chosen complement $\mathcal{V}$ in the sense that on invariant sub-Riemannian structures on principal bundles, Ric $\mathcal{H \mathcal { V }}$ measures how far $\mathcal{H}$ is from being a Yang-Mills connection, see Example 4.3. We will see how Ric $\mathcal{H}$ V can be interpreted in a similar way in the general case in Appendix A.4, Part II.

For further geometric interpretation, see Part II, Section 5.2.

Remark 3.6. In the proof of Theorem 3.5. we prove a curvature-dimension inequality without normalizing $\mathscr{M}_{\mathcal{R}}$ in (3.9). The reason why we are free to normalize $\mathbf{v}$ such that $\mathscr{M}_{\mathcal{R}}=1$ is the following. Since $\nabla_{A} Z$ is independent of $\mathbf{v}$ when either $A$ or $Z$ are horizontal, the bounds introduced in (i)-(v) behave well under scaling in the sense that for any $\varepsilon>0$, if we define the bounds relative to $\mathbf{v}_{2}=\frac{1}{\varepsilon} \mathbf{v}$ rather than $\mathbf{v}$, we will get the same inequality back for $\Gamma_{2}^{\mathbf{h}^{*}}(f)+\frac{\ell}{\varepsilon} \Gamma_{2}^{\mathbf{v}_{2}^{*}}(f)=\Gamma_{2}^{\mathbf{h}^{*}}(f)+\ell \Gamma_{2}^{\mathbf{v}^{*}}(f)$.

Remark 3.7. In parallel with the development of our paper, Theorem 3.5 for the case $\stackrel{\circ}{\nabla}^{*}=0, \mathscr{M}_{\mathcal{H V}}=0$ appeared in $[9]$.

3.3. Totally geodesic foliations. Let $(M, \mathcal{H}, \mathbf{h})$ be a sub-Riemannian manifold with an integrable, metric-preserving complement $\mathcal{V}$. Let $\mathbf{v}$ be a chosen metric on $\mathcal{V}$ and assume that $\stackrel{\circ}{\nabla}^{*}=0$. By Section 2.4 if vol is the volume form of the Riemannian metric $\mathbf{g}$ corresponding to $\mathbf{v}$, then $\Delta_{\mathbf{h}}^{\prime}$ coincides with the subLaplacian $\Delta_{\mathbf{h}}$ defined relative to vol. By Theorem 3.5 we also obtain a somewhat 
simpler curvature-dimension inequality

$$
\begin{aligned}
& \Gamma_{2}^{\mathbf{h}^{*}+\ell \mathbf{v}^{*}}(f) \geq \frac{1}{n}(L f)^{2}+\left(\rho_{1}-\frac{1}{\ell}\right) \Gamma^{\mathbf{h}^{*}}(f)+\rho_{2} \Gamma^{\mathbf{v}^{*}}(f), \\
& n=\operatorname{rank} \mathcal{H}, \quad \rho_{1}=\rho_{\mathcal{H}}-c^{-1}, \quad \rho_{2}=\frac{1}{2} m_{\mathcal{R}}^{2}-c \mathscr{M}_{\mathcal{H} \mathcal{V}}^{2},
\end{aligned}
$$

where $c>0$ is arbitrary. The inequality (CD) with the additional assumption $\rho_{2}>0$ was originally suggested as a generalization of the curvature-dimension inequality by Baudoin and Garofalo 8 .

We will also need the following relation, which is closely related to the inequality (CD). The proof is left to Section 3.6. This result is essential for proving the result of Theorem 5.1 (b).

Proposition 3.8. For any $f \in C^{\infty}(M)$, and any $c>0$ and $\ell>0$, we have

$$
\begin{aligned}
& \frac{1}{4} \Gamma^{\mathbf{h}^{*}}\left(\Gamma^{\mathbf{h}^{*}}(f)\right) \leq \Gamma^{\mathbf{h}^{*}}(f)\left(\Gamma_{2}^{\mathbf{h}^{*}+\ell \mathbf{v}^{*}}(f)-\left(\varrho_{1}-\ell^{-1}\right) \Gamma^{\mathbf{h}^{*}}(f)-\varrho_{2} \Gamma^{\mathbf{v}^{*}}(f)\right), \\
& \frac{1}{4} \Gamma^{\mathbf{h}^{*}}\left(\Gamma^{\mathbf{v}^{*}}(f)\right) \leq \Gamma^{\mathbf{v}^{*}}(f) \Gamma_{2}^{\mathbf{v}^{*}}(f),
\end{aligned}
$$

where $\varrho_{1}=\rho_{\mathcal{H}}-c^{-1}$ and $\varrho_{2}=-c \mathscr{M}_{\mathcal{H} \mathcal{V}}^{2}$.

Remark 3.9. To give some context for Proposition 3.8, consider the following special case. Let $\mathbf{h}=\mathbf{g}$ be a complete Riemannian metric on $M$ with lower Ricci bound $\rho$ an choose $\mathbf{v}^{*}=0$. Inserting this in (CD with $\ell=\infty$ gives us $\Gamma_{2}^{\mathbf{g}^{*}}(f) \geq \frac{1}{n}(\Delta f)+\rho \Gamma^{\mathbf{g}^{*}}(f)$ where $\Delta$ is the Laplacian of $\mathbf{g}$. If we let $P_{t}=e^{t \Delta / 2}$ be the heat semigroup of $\frac{1}{2} \Delta$, then the previously mentioned inequality implies the inequality $\Gamma^{\mathrm{g}^{*}}\left(P_{t} f\right) \leq e^{-\rho t} P_{t} \Gamma^{\mathrm{g}^{*}}(f)$ for any smooth, compactly supported function $f$. However, Proposition 3.8 gives us $\Gamma^{\mathbf{g}^{*}}\left(\Gamma^{\mathbf{g}^{*}}(f)\right) \leq 4 \Gamma^{\mathbf{g}^{*}}(f)\left(\Gamma_{2}^{\mathbf{g}^{*}}(f)-\rho \Gamma^{\mathbf{g}^{*}}(f)\right)$ which imply the stronger result $\Gamma^{\mathbf{g}^{*}}\left(P_{t} f\right)^{1 / 2} \leq e^{-\rho / 2 t} P_{t}\left(\Gamma^{\mathbf{g}^{*}}(f)^{1 / 2}\right)$ for any smooth, compactly supported function $f$, see e.g. [4, Section 2].

Remark 3.10. If a metric $\mathbf{v}$ on $\mathcal{V}$ exist with ${ }^{\circ} \mathbf{v}=0$, then it is uniquely determined by its value at one point. To see this, let $\mathbf{v}^{\prime}$ be an arbitrary metric on $\mathcal{V}$ and let $\gamma$ be a horizontal curve in $M$. Define $\nabla^{\prime}$ with respect to $\mathbf{v}^{\prime}$. By Lemma 3.2 (a), we still have $\nabla_{\dot{\gamma}}^{\prime} \mathbf{v}=0$. Since $\mathcal{H}$ is bracket-generating, the value of $\mathbf{v}$ at any point can be determined by parallel transport along a horizontal curve from one given point.

3.4. A convenient choice of bases for $\mathcal{H}$ and $\mathcal{V}$. Let $(M, \mathcal{H}, \mathbf{h})$ be a subRiemannian manifold with an integrable metric-preserving complement of $\mathcal{V}$. Let $\mathbf{v}$ be a metric tensor on $\mathcal{V}$. To simplify the proof of Theorem 3.5, we first want to introduce a convenient choice of bases for $\mathcal{H}$ and $\mathcal{V}$ that will simplify our calculations, similar to choosing the coordinate vector fields of a normal coordinate system in Riemannian geometry. Let $\nabla$ be defined as in (2.6).

Lemma 3.11. Given an arbitrary point $x_{0}$ of $M$, there are local orthonormal bases $A_{1}, \ldots, A_{n}$ and $V_{1}, \ldots, V_{\nu}$ of respectively $\mathcal{H}$ and $\mathcal{V}$ defined in a neighborhood around $x_{0}$ such that for any vector field $Z$,

$$
\left.\stackrel{\infty}{\nabla}_{Z} A_{i}\right|_{x_{0}}=\left.\nabla_{Z}^{\infty} V_{s}\right|_{x_{0}}=0 .
$$

In particular, these bases have the properties

$$
\left.\operatorname{pr}_{\mathcal{H}}\left[A_{i_{1}}, A_{i_{2}}\right]\right|_{x_{0}}=0,\left.\quad \operatorname{pr}_{\mathcal{V}}\left[V_{s_{1}}, V_{s_{2}}\right]\right|_{x_{0}}=0
$$


Proof. Define a Riemannian metric $\mathbf{g}$ by $\mathbf{g}=\operatorname{pr}_{\mathcal{H}}^{*} \mathbf{h}+\operatorname{pr}_{\mathcal{V}}^{*} \mathbf{v}$. Let $\left(x_{1}, \ldots, x_{n+\nu}\right)$ be a normal coordinate system relative to $\mathbf{g}$ centered at $x_{0}$ such that

$$
\mathcal{H}_{x_{0}}=\operatorname{span}\left\{\left.\frac{\partial}{\partial x_{1}}\right|_{x_{0}}, \ldots,\left.\frac{\partial}{\partial x_{n}}\right|_{x_{0}}\right\}, \quad \mathcal{V}_{x_{0}}=\operatorname{span}\left\{\left.\frac{\partial}{\partial x_{n+1}}\right|_{x_{0}}, \ldots,\left.\frac{\partial}{\partial x_{n+\nu}}\right|_{x_{0}}\right\} \text {. }
$$

Define $Y_{j}=\operatorname{pr}_{\mathcal{H}} \frac{\partial}{\partial x_{j}}$ and $Z_{j}=\operatorname{pr}_{\mathcal{V}} \frac{\partial}{\partial x_{n+j}}$. These vector fields are linearly independent close to $x_{0}$. Write

$$
Y_{j}=\sum_{i=1}^{n+\nu} a_{i j} \frac{\partial}{\partial x_{i}}, \quad Z_{s}=\sum_{i=1}^{n+\nu} b_{i s} \frac{\partial}{\partial x_{i}}
$$

where

$$
a_{i j}\left(x_{0}\right)=\left\{\begin{array}{ll}
1 & \text { if } i=j \\
0 & \text { if } i \neq j
\end{array}, \quad b_{i s}\left(x_{0}\right)= \begin{cases}1 & \text { if } i=s+n \\
0 & \text { if } i \neq s+n\end{cases}\right.
$$

and consider the matrix-valued functions

$$
a=\left(a_{i j}\right)_{i, j=1}^{n}, \quad b=\left(b_{n+r, n+s}\right)_{r, s=1}^{\nu} .
$$

These matrices remain invertible in a neighborhood of $x_{0}$. On this mentioned neighborhood, let $\alpha=\left(\alpha_{i j}\right)=a^{-1}$ and $\beta=\left(\beta_{r s}\right)=b^{-1}$. Define $\tilde{Y}_{j}=\sum_{i=1}^{n} \alpha_{i j} Y_{i}$ and $\widetilde{Z}_{s}=\sum_{r=1}^{\nu} \beta_{r s} Z_{r}$. These bases can then we written in the form

$$
\widetilde{Y}_{j}=\frac{\partial}{\partial x_{j}}+\sum_{i=n+1}^{n+\nu} \widetilde{a}_{i j} \frac{\partial}{\partial x_{i}}, \quad \widetilde{Z}_{j}=\frac{\partial}{\partial x_{n+j}}+\sum_{i=1}^{n} \widetilde{b}_{i j} \frac{\partial}{\partial x_{i}},
$$

for some functions $\widetilde{a}_{i j}$ and $\widetilde{b}_{i j}$ which vanish at $x_{0}$. These bases clearly satisfy (3.5) and (3.6).

Since $\nabla$ preserves the metric, we can use the Gram-Schmidt process to obtain $A_{1}, \ldots, A_{n}$ and $V_{1}, \ldots V_{\nu}$ from respectively $\widetilde{Y}_{1}, \ldots, \widetilde{Y}_{\nu}$ and $\widetilde{Z}_{1}, \ldots, \widetilde{Z}_{\nu}$.

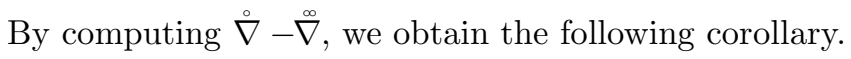

Corollary 3.12. Given an arbitrary point $x_{0}$ of $M$, then around $x_{0}$ there are local orthonormal bases $A_{1}, \ldots, A_{n}$ and $V_{1}, \ldots, V_{\nu}$ of respectively $\mathcal{H}$ and $\mathcal{V}$ such that for any vector field $Z$,

$$
\begin{aligned}
& \left.\stackrel{\circ}{\nabla}_{Z} A_{i}\right|_{x_{0}}=\left.\frac{1}{2} \sharp \mathbf{g}\left(Z, \mathcal{R}\left(A_{i}, \cdot\right)\right)\right|_{x_{0}}, \\
& \left.\stackrel{\circ}{\nabla}_{Z} V_{s}\right|_{x_{0}}=-\left.\frac{1}{2} \sharp\left(\stackrel{\circ}{\nabla}_{Y} \mathbf{g}\right)\left(V_{s}, \cdot\right)\right|_{x_{0}},
\end{aligned}
$$

where $\sharp: T^{*} M \rightarrow T M$ is the identification defined relative to $\mathrm{g}$. In particular, these bases have the properties $\left.\operatorname{pr}_{\mathcal{H}}\left[A_{i_{1}}, A_{i_{2}}\right]\right|_{x_{0}}=0$, and $\left.\operatorname{pr}_{\mathcal{V}}\left[V_{s_{1}}, V_{s_{2}}\right]\right|_{x_{0}}=0$.

3.5. Proof of Theorem 3.5. Let $\mathbf{h}$ and $\mathbf{v}$ be the respective metrics on $\mathcal{H}$ and $\mathcal{V}$ that give us a Riemannian metric $\mathbf{g}=\operatorname{pr}_{\mathcal{H}}^{*} \mathbf{h}+\operatorname{pr}_{\mathcal{V}}^{*} \mathbf{v}$. Let $b: T M \rightarrow T^{*} M$ be the map $v \mapsto \mathbf{g}(v,$.$) with inverse \sharp$. Let $\sharp \mathbf{v}^{*}$ be defined similar to the definition of $\sharp^{*}$ in Section 2.1. Note that $\sharp \mathbf{h}^{*}=\operatorname{pr}_{\mathcal{H}} \sharp$ and $\sharp \mathbf{v}^{*}=\operatorname{pr}_{\mathcal{V}} \sharp$.

Let $A_{1}, \ldots, A_{n}$ be as in Corollary 3.12 relative to some point $x_{0}$. Clearly, for any $f \in C^{\infty}(M)$, we have $L f\left(x_{0}\right)=\sum_{i=1}^{n} A_{i}^{2} f\left(x_{0}\right)$. Note also that

$$
\stackrel{\circ}{\nabla}_{A} d f(Z)=\stackrel{\circ}{\nabla}_{Z} d f(A)-d f\left(T^{\nabla^{\nabla}}(A, Z)\right)=\stackrel{\circ}{\nabla}_{Z} d f(A)+d f(\mathcal{R}(A, Z)) .
$$


In the following calculations, since $\mathcal{V}$ is metric-preserving, keep in mind that

$$
\stackrel{\circ}{\nabla}_{A} \sharp^{\mathbf{h}^{*}} d f=\sharp^{\mathbf{h}^{*}} \stackrel{\circ}{\nabla}_{A} d f,
$$

while $\stackrel{\circ}{\nabla}_{A} \sharp^{*} d f=\sharp^{*} \stackrel{\circ}{\nabla}_{A} d f+\left(\stackrel{\circ}{\nabla}_{A} \mathbf{v}^{*}\right)(d f,$.$) ,.$

Below, all terms are evaluated at $x_{0}$. We first note that for any $\ell>0$,

$$
\begin{aligned}
\Gamma_{2}^{\mathbf{h}^{*}+\ell \mathbf{v}^{*}}(f)= & \frac{1}{2} \sum_{i=1}^{n} A_{i}^{2}\left(\|d f\|_{\mathbf{h}^{*}}^{2}+\ell\|d f\|_{\mathbf{v}^{*}}^{2}\right)-\mathbf{h}^{*}(d f, d L f)-\ell \mathbf{v}^{*}(d f, d L f) \\
= & \sum_{i=1}^{n} A_{i} \stackrel{\circ}{\nabla}_{A_{i}} d f\left(\sharp^{\mathbf{h}^{*}} d f\right)+\ell \sum_{i=1}^{n} A_{i} \stackrel{\circ}{\nabla}_{A_{i}} d f\left(\sharp \mathbf{v}^{*} d f\right) \\
& +\frac{1}{2} \ell \sum_{i=1}^{n} A_{i}\left(\stackrel{\circ}{\nabla}_{A_{i}} \mathbf{v}^{*}\right)(d f, d f)-\left(\sharp^{\mathbf{h}^{*}} d f+\ell \sharp^{\mathbf{v}^{*}} d f\right)\left(\sum_{i=1}^{n} \stackrel{\circ}{\nabla}_{A_{i}} d f\left(A_{i}\right)\right) \\
= & \sum_{i=1}^{n} \stackrel{\circ}{\nabla}_{A_{i}} \stackrel{\circ}{\nabla}_{\sharp \mathbf{h}^{*}} d f d f\left(A_{i}\right)+\sum_{i=1}^{n} A_{i} d f\left(\mathcal{R}\left(A_{i}, \sharp^{\mathbf{h}^{*}} d f\right)\right) \\
& +\ell \sum_{i=1}^{n} \stackrel{\circ}{\nabla}_{A_{i}} \stackrel{\circ}{\sharp}_{\sharp \mathbf{v}^{*}} d f d f\left(A_{i}\right)-\sum_{i=1}^{n} \stackrel{\circ}{\sharp}_{\mathbf{h}^{*}} \stackrel{\circ}{\nabla}_{A_{i}} d f\left(A_{i}\right) \\
& -\ell \sum_{i=1}^{n} \stackrel{\circ}{\nabla}_{\sharp \mathbf{v}^{*}} \stackrel{\circ}{\nabla}_{A_{i}} d f\left(A_{i}\right)-\frac{1}{2} \ell \sum_{i=1}^{n} d f\left(\mathcal{R}\left(A_{i}, \sharp^{\mathbf{h}^{*}} \stackrel{\circ}{\nabla}_{A_{i}} d f\right)\right) \\
& +\frac{1}{2} \ell\left(\Delta_{\mathbf{h}}^{\prime} \mathbf{v}^{*}\right)(d f, d f)+\ell \sum_{i=1}^{n}\left(\stackrel{\circ}{\nabla}_{A_{i}} \mathbf{v}^{*}\right)\left(\stackrel{\circ}{\nabla}_{A_{i}} d f, d f\right) .
\end{aligned}
$$

Observe that

$$
\begin{aligned}
& \stackrel{\circ}{\nabla}_{A_{i}} \stackrel{\circ}{\nabla}_{\sharp \mathrm{h}^{*}} d f d f\left(A_{i}\right)-\stackrel{\circ}{\nabla}_{\sharp \mathrm{h}^{*}} d f \stackrel{\circ}{\nabla}_{A_{i}} d f\left(A_{i}\right) \\
& \left.=\sum_{i=1}^{n} \mathbf{g}\left(R^{\stackrel{\circ}{\nabla}}\left(A_{i}, \sharp^{\mathbf{h}^{*}} d f\right) \sharp^{\mathbf{h}^{*}} d f, A_{i}\right)+\sum_{i=1}^{n} \stackrel{\circ}{\nabla}_{\left[A_{i}, \sharp \mathbf{h}^{*}\right.} d f\right] d f\left(A_{i}\right) \\
& \left.=\operatorname{Ric}_{\mathcal{H}}\left(\sharp^{\mathbf{h}^{*}} d f, \sharp^{\mathbf{h}^{*}} d f\right)+\sum_{i=1}^{n} \stackrel{\circ}{\nabla}_{\stackrel{\triangleright}{\nabla}_{A_{i}} \sharp \mathbf{h}^{*} d f} d f\left(A_{i}\right)+\sum_{i=1}^{n} \stackrel{\circ}{\nabla}_{\mathcal{R}\left(A_{i}, \sharp \mathbf{h}^{*}\right.} d f\right) d f\left(A_{i}\right) \\
& =\operatorname{Ric} \mathcal{H}\left(\sharp^{\mathbf{h}^{*}} d f, \sharp^{\mathbf{h}^{*}} d f\right)+\sum_{i=1}^{n}\left\|\stackrel{\circ}{\nabla}_{A_{i}} d f\right\|_{\mathbf{h}^{*}}^{2} \\
& -\sum_{i=1}^{n} d f\left(\mathcal{R}\left(A_{i}, \sharp^{\mathbf{h}^{*}} \stackrel{\circ}{\nabla}_{A_{i}} d f\right)\right)+\sum_{i=1}^{n} \stackrel{\circ}{\nabla}_{A_{i}} d f\left(\mathcal{R}\left(A_{i}, \sharp^{\mathbf{h}^{*}} d f\right)\right),
\end{aligned}
$$

while

$$
\begin{aligned}
& \stackrel{\circ}{\nabla}_{A_{i}}{\stackrel{\circ}{\nabla^{*} \mathbf{v}^{*}}}_{d f} d f\left(A_{i}\right)-\stackrel{\circ}{\nabla}_{\sharp \mathbf{v}^{*}} d f \stackrel{\circ}{\nabla}_{A_{i}} d f\left(A_{i}\right) \\
& =\sum_{i=1}^{n} \mathbf{g}\left(R^{\stackrel{\circ}{\nabla}}\left(A_{i}, \sharp^{*} d f\right) \sharp^{*} \mathbf{h}^{*} d f, A_{i}\right) \\
& +\sum_{i=1}^{n} \stackrel{\circ}{\nabla}_{\dot{\nabla}_{A_{i}} \sharp \mathbb{v}^{*} d f} d f\left(A_{i}\right)-\sum_{i=1}^{n} \stackrel{\circ}{\nabla}_{\dot{\nabla}_{\sharp \mathbf{v}^{*} d f}} A_{i} d f\left(A_{i}\right)
\end{aligned}
$$




$$
\begin{aligned}
= & \sum_{i=1}^{n} \stackrel{\circ}{\nabla}_{\sharp \mathbf{v}^{*} \circ^{\circ}}{ }_{A_{i}} d f+\left(\stackrel{\circ}{\nabla}_{A_{i}} \mathbf{v}^{*}\right)(d f, \cdot \boldsymbol{\cdot}) \\
= & \sum_{i=1}^{n}\left\|\stackrel{\circ}{\nabla}_{A_{i}} d f\right\|_{\mathbf{v}^{*}}^{2}+\sum_{i=1}^{n}\left(\stackrel{\circ}{\nabla}_{A_{i}} \mathbf{v}^{*}\right)\left(d f, \stackrel{\circ}{\nabla}_{A_{i}} d f\right) \\
& -\frac{1}{2} \sum_{i=1}^{n} d f\left(\mathcal{R}\left(A_{i}, \sharp^{\mathbf{h}^{*}} \stackrel{\circ}{\nabla}_{A_{i}} d f\right)\right)+\|d f(\mathcal{R}(\cdot, \cdot))\|_{\wedge^{2}}^{2} \mathbf{g}^{*} \cdot
\end{aligned}
$$

Hence

$$
\begin{aligned}
\Gamma_{2}^{\mathbf{h}^{*}+\ell \mathbf{v}^{*}}(f)= & \sum_{i=1}^{n}\left\|\stackrel{\circ}{\nabla}_{A_{i}} d f\right\|_{\mathbf{h}^{*}}^{2}+\operatorname{Ric}_{\mathcal{H}}\left(\sharp^{\mathbf{h}^{*}} d f, \sharp^{\mathbf{h}^{*}} d f\right)-\sum_{i=1}^{n} d f\left(\mathcal{R}\left(A_{i}, \sharp^{*} \stackrel{\circ}{\nabla}_{A_{i}} d f\right)\right) \\
& +\sum_{i=1}^{n} \stackrel{\circ}{\nabla}_{A_{i}} d f\left(\mathcal{R}\left(A_{i}, \sharp^{\mathbf{h}^{*}} d f\right)\right)+\sum_{i=1}^{n} A_{i} d f\left(\mathcal{R}\left(A_{i}, \sharp^{*} d f\right)\right) \\
& +\ell \sum_{i=1}^{n}\left\|\stackrel{\circ}{\nabla}_{A_{i}} d f\right\|_{\mathbf{v}^{*}}^{2}+\ell \sum_{i=1}^{n}\left(\stackrel{\circ}{\nabla}_{A_{i}} \mathbf{v}^{*}\right)\left(d f, \stackrel{\circ}{\nabla}_{A_{i}} d f\right) \\
& -\ell \sum_{i=1}^{n} d f\left(\mathcal{R}\left(A_{i}, \sharp \mathbf{h}^{*} \stackrel{\circ}{\nabla}_{A_{i}} d f\right)\right)+\ell\|d f(\mathcal{R}(\cdot, \cdot))\|_{\wedge^{2} \mathbf{g}^{*}}^{2} \\
& +\frac{1}{2} \ell\left(\Delta_{\mathbf{h}}^{\prime} \mathbf{v}^{*}\right)(d f, d f)+\ell \sum_{i=1}^{n}\left(\stackrel{\circ}{\nabla}_{A_{i}} \mathbf{v}^{*}\right)\left(\stackrel{\circ}{\nabla}_{A_{i}} d f, d f\right) .
\end{aligned}
$$

By realizing that

$$
\begin{aligned}
\sum_{i=1}^{n} A_{i} d f\left(\mathcal{R}\left(A_{i}, \sharp \mathbf{h}^{*} d f\right)\right)= & \operatorname{Ric}_{\mathcal{H} \mathcal{V}}(\sharp d f, \sharp d f)+\sum_{i=1}^{n} \stackrel{\circ}{\nabla}_{A_{i}} d f\left(\mathcal{R}\left(A_{i}, \sharp \mathbf{h}^{*} d f\right)\right) \\
& +\sum_{i=1}^{n} d f\left(\mathcal{R}\left(A_{i}, \sharp^{*} \stackrel{\circ}{\nabla}_{A_{i}} d f\right)\right),
\end{aligned}
$$

and that

we obtain

$$
d f\left(\mathcal{R}\left(A_{i}, \sharp^{\mathbf{h}^{*}} \stackrel{\circ}{\nabla}_{A_{i}} d f\right)\right)=\|d f(\mathcal{R}(\cdot, \cdot))\|_{\wedge^{2} \mathbf{g}^{*}}^{2},
$$

$$
\begin{aligned}
\Gamma_{2}^{\mathbf{h}^{*}+\ell \mathbf{v}^{*}}(f)= & \sum_{i=1}^{n}\left\|\stackrel{\circ}{\nabla}_{A_{i}} d f\right\|_{\mathbf{h}^{*}}^{2}+\operatorname{Ric}_{\mathcal{H}}\left(\sharp^{\mathbf{h}^{*}} d f, \sharp^{\mathbf{h}^{*}} d f\right) \\
& +\operatorname{Ric} \mathcal{H} \mathcal{V}(\sharp d f, \sharp d f)+2 \sum_{i=1}^{n} \stackrel{\circ}{\nabla}_{A_{i}} d f\left(\mathcal{R}\left(A_{i}, \sharp^{\mathbf{h}^{*}} d f\right)\right) \\
& +\ell \sum_{i=1}^{n}\left\|\stackrel{\circ}{\nabla}_{A_{i}} d f\right\|_{\mathbf{v}^{*}}^{2}+2 \ell \sum_{i=1}^{n}\left(\stackrel{\circ}{\nabla}_{A_{i}} \mathbf{v}^{*}\right)\left(d f, \stackrel{\circ}{\nabla}_{A_{i}} d f\right) \\
& +\frac{1}{2} \ell\left(\Delta_{\mathbf{h}}^{\prime} \mathbf{v}^{*}\right)(d f, d f) .
\end{aligned}
$$

Clearly

$$
\ell \sum_{i=1}^{n}\left\|\stackrel{\circ}{\nabla}_{A_{i}} d f\right\|_{\mathbf{v}^{*}}^{2}+2 \stackrel{\circ}{\nabla}_{A_{i}} d f\left(\mathcal{R}\left(A_{i}, \sharp^{\mathbf{h}^{*}} d f\right)\right)+2 \ell \sum_{i=1}^{n}\left(\stackrel{\circ}{\nabla}_{A_{i}} \mathbf{v}^{*}\right)\left(d f, \stackrel{\circ}{\nabla}_{A_{i}} d f\right)
$$




$$
\begin{aligned}
& \geq-\frac{1}{\ell} \sum_{i=1}^{n}\left\|\ell\left(\stackrel{\circ}{\nabla}_{A_{i}} \mathbf{v}^{*}\right)(d f, .)+\mathcal{R}\left(A_{i}, \sharp \mathbf{h}^{*} d f\right)\right\|_{\mathbf{v}}^{2} \\
& \geq-\frac{\mathscr{M}_{\mathcal{R}}^{2}}{\ell} \Gamma^{\mathbf{h}^{*}}(f)-2 \mathscr{M}_{\mathcal{R}} \mathscr{M}_{\dot{\nabla} \mathbf{v}^{*}} \sqrt{\Gamma \mathbf{h}^{*}(f) \Gamma \mathbf{v}^{*}(f)}-\ell \mathscr{M}_{\dot{\nabla} \mathbf{v}^{*}}^{2} \Gamma^{\mathbf{v}^{*}}(f),
\end{aligned}
$$

and also

$$
\begin{aligned}
& \sum_{i=1}^{n}\left\|\stackrel{\circ}{\nabla}_{A_{i}} d f\right\|_{\mathbf{h}^{*}}^{2}=\sum_{i, j=1}^{n}\left(\frac{1}{2}\left(\stackrel{\circ}{\nabla}_{A_{i}, A_{j}}^{2} f+\stackrel{\circ}{\nabla}_{A_{j}, A_{i}}^{2} f\right)+\frac{1}{2}\left(\stackrel{\circ}{\nabla}_{A_{i}, A_{j}} f-\stackrel{\circ}{\nabla}_{A_{i}, A_{j}} f\right)\right)^{2} \\
& =\sum_{i, j=1}^{n}\left(\frac{1}{2}\left(\stackrel{\circ}{\nabla}_{A_{i}, A_{j}}^{2} f+\stackrel{\circ}{\nabla}_{A_{j}, A_{i}}^{2} f\right)\right)^{2}+\sum_{i, j=1}^{n}\left(\frac{1}{2}\left(\stackrel{\circ}{\nabla}_{A_{i}, A_{j}} f-\stackrel{\circ}{\nabla}_{A_{i}, A_{j}} f\right)\right)^{2} \\
& \geq \sum_{i=1}^{n}\left(\stackrel{\circ}{\nabla}_{A_{i}, A_{i}}^{2} f\right)^{2}+\sum_{i, j=1}^{n}\left(\frac{1}{2} d f\left(\mathcal{R}\left(A_{i}, A_{j}\right)\right)\right)^{2} \geq \frac{1}{n}(L f)^{2}+\frac{1}{2} m_{\mathcal{R}}^{2} \Gamma^{\mathbf{v}^{*}}(f) .
\end{aligned}
$$

In conclusion

$$
\begin{aligned}
\Gamma_{2}^{\mathbf{h}^{*}+\ell \mathbf{v}^{*}}(f) \geq & \frac{1}{n}(L f)^{2}+\left(\rho_{\mathcal{H}}-\frac{\mathscr{M}_{\mathcal{R}}^{2}}{\ell}\right) \Gamma^{\mathbf{h}^{*}}(f) \\
& -\left(2 \mathscr{M}_{\mathcal{H} \mathcal{V}}+2 \mathscr{M}_{\mathcal{R}} \mathscr{M}_{\nabla \mathbf{v}^{*}}\right) \sqrt{\Gamma \mathbf{h}^{*}(f) \Gamma^{\mathbf{v}^{*}}(f)} \\
& +\frac{1}{2}\left(m_{\mathcal{R}}^{2}+\ell\left(\rho_{\Delta_{\mathbf{h}}^{\prime} \mathbf{v}^{*}}-2 \mathscr{M}_{\nabla \mathbf{v}^{*}}^{2}\right)\right) \Gamma^{\mathbf{v}^{*}}(f) \\
\geq & \frac{1}{n}(L f)^{2}+\left(\rho_{\mathcal{H}}-\frac{1}{c}-\frac{\mathscr{M}_{\mathcal{R}}^{2}}{\ell}\right) \Gamma^{\mathbf{h}^{*}}(f) \\
& +\frac{1}{2}\left(m_{\mathcal{R}}^{2}-2 c\left(\mathscr{M}_{\mathcal{H} \mathcal{V}}+\mathscr{M}_{\mathcal{R}} \mathscr{M}_{\nabla \mathbf{v}^{*}}\right)^{2}\right) \Gamma^{\mathbf{v}^{*}}(f) \\
& +\ell\left(\rho_{\Delta_{\mathbf{h}}^{\prime} \mathbf{v}^{*}}-2 \mathscr{M}_{\nabla \mathbf{v}^{*}}^{2}\right) \Gamma^{\mathbf{v}^{*}}(f) .
\end{aligned}
$$

3.6. Proof of Proposition 3.8. Let $A_{1}, \ldots, A_{n}$ be a local orthonormal basis of $\mathcal{H}$. From the assumption $\stackrel{\circ}{\nabla}^{*}=0$ and (3.8), we obtain $\Gamma_{2}^{\mathbf{v}^{*}}(f)=\sum_{i=1}^{n}\left\|\nabla^{\circ} A_{i} d f\right\|_{\mathbf{v}^{*}}^{2}$ and from this, we know

$$
\begin{aligned}
\Gamma^{\mathbf{h}^{*}}\left(\Gamma^{\mathbf{v}^{*}}(f)\right) & =4 \sum_{i=1}^{n} \mathbf{v}^{*}\left(\stackrel{\circ}{\nabla}_{A_{i}} d f, d f\right)^{2} \\
& \leq 4 \sum_{i=1}^{n}\left\|\nabla_{A_{i}} d f\right\|_{\mathbf{v}^{*}}^{2}\|d f\|_{\mathbf{v}^{*}}=4 \Gamma_{2}^{\mathbf{v}^{*}}(f) \Gamma^{\mathbf{v}^{*}}(f) .
\end{aligned}
$$

Similarly, from (3.8),

$$
\begin{aligned}
\sum_{i=1}^{n}\left\|\stackrel{\circ}{\nabla}_{A_{i}} d f\right\|_{\mathbf{h}^{*}}^{2}= & \Gamma_{2}^{\mathbf{h}^{*}+\ell \mathbf{v}^{*}}(f)-\operatorname{Ric}_{\mathcal{H}}\left(\sharp^{\mathbf{h}^{*}} d f, \sharp^{\mathbf{h}^{*}} d f\right)-2 \operatorname{Ric} \mathcal{H} \mathcal{V}\left(\sharp^{\mathbf{v}^{*}} d f, \sharp^{\mathbf{h}^{*}} d f\right) \\
& -2 \sum_{i=1}^{n} \stackrel{\circ}{\nabla}_{A_{i}} d f\left(\mathcal{R}\left(A_{i}, \sharp^{\mathbf{h}^{*}} d f\right)\right)+\ell \sum_{i=1}^{n}\left\|\stackrel{\circ}{\nabla}_{A_{i}} d f\right\|_{\mathbf{v}^{*}}^{2} \\
\leq & \Gamma_{2}^{\mathbf{h}+\ell \mathbf{v}^{*}}(f)-\left(\rho_{\mathcal{H}}-c^{-1}-\ell^{-1}\right) \Gamma^{\mathbf{h}^{*}}(f)+c \mathscr{M}_{\mathcal{H} \mathcal{V}}^{2} \Gamma^{\mathbf{v}^{*}}(f),
\end{aligned}
$$

and the result follows. 
3.7. For a general choice of $L$. Let $(M, \mathcal{H}, \mathbf{h})$ and $\mathcal{V}$ be as in Section 3.2 and let $\Delta_{\mathbf{h}}^{\prime}$ be the sub-Laplacian of $\mathcal{V}$. For a general choice of $L$ with $\mathbf{q}_{L}=\mathbf{h}^{*}$, write $L=\Delta_{\mathbf{h}}^{\prime}+Z$ for some vector field $Z$. We want to use our generalized curvaturedimension inequality for $\Delta_{\mathbf{h}}^{\prime}$ to extend it to a more general class of operators. Unfortunately, our possibilities are somewhat limited.

Proposition 3.13. Let $L=\Delta_{\mathbf{h}}^{\prime}+Z$ where $Z \in \Gamma(\mathcal{V})$ is a non-zero vertical vector field. Then $L$ satisfies (CD*) with $\operatorname{rank} \mathcal{H}<n \leq \infty$ and $\rho_{1}, \rho_{2,0}, \rho_{2,1}$ given by

$$
\begin{aligned}
\rho_{1} & =\rho_{\mathcal{H}}-c^{-1}, \\
\rho_{2,0} & =\frac{1}{2} m_{\mathcal{R}}^{2}-c\left(\mathscr{M}_{\mathcal{H} \mathcal{V}}^{Z}+\mathscr{M}_{\nabla^{*} \mathbf{v}^{*}}\right)^{2}-\frac{1}{n-\operatorname{rank} \mathcal{H}}\|Z\|_{\mathbf{v}^{*}}^{2}, \\
\rho_{2,1} & =\frac{1}{2} \rho_{\Delta_{\mathbf{h}}^{\prime} \mathbf{v}^{*}}-\mathscr{M}_{\nabla_{\mathbf{v}}^{*}}^{2}-\mathscr{N}^{2},
\end{aligned}
$$

for any positive $c>0$. Here, $-\mathscr{M}_{\mathcal{H V}}^{Z}$ is a lower bound of

$$
\begin{aligned}
\operatorname{Ric}_{\mathcal{H} \mathcal{V}}^{Z}\left(A_{1}, A_{2}\right):= & \operatorname{Ric}_{\mathcal{H} \mathcal{V}}\left(A_{1}, A_{2}\right)+\frac{1}{2} \mathbf{h}\left(\operatorname{pr}_{\mathcal{H}} A_{1}, \stackrel{\circ}{\nabla}_{\operatorname{pr}_{\mathcal{H}} A_{2}} Z\right)+\frac{1}{2} \mathbf{h}\left(\operatorname{pr}_{\mathcal{H}} A_{2}, \stackrel{\circ}{\nabla}_{\operatorname{pr}_{\mathcal{H}} A_{1}} Z\right) \\
& +\frac{1}{2} \mathbf{v}\left(\operatorname{pr}_{\mathcal{V}} A_{1}, \mathcal{R}\left(Z, A_{2}\right)\right)+\frac{1}{2} \mathbf{v}\left(\operatorname{pr}_{\mathcal{V}} A_{2}, \mathcal{R}\left(Z, A_{1}\right)\right)
\end{aligned}
$$

and $\mathscr{N}$ is a lower bound of $\mathbf{v}\left(\operatorname{pr}_{\mathcal{V}} \cdot, \stackrel{\circ}{\nabla}_{\mathrm{pr}_{\mathcal{V}}}, Z\right)$. The other constants are as in Section 3.2 .

Proof. Let $\sharp \mathbf{h}^{*}$ be defined as in Section 2.1 and let $\sharp^{*}$ be defined analogously. Then the result follows from the identities,

$$
\begin{aligned}
\frac{1}{2} Z \Gamma^{\mathbf{h}^{*}}(f)-\Gamma^{\mathbf{h}^{*}}(Z f, f) & =d f\left({\stackrel{\circ}{\mathbf{H}^{*}}}^{*} d f\right. \\
\frac{1}{2} Z \Gamma^{\mathbf{v}^{*}}(f)-\Gamma^{\mathbf{v}^{*}}(Z f, f) & =d f\left(\stackrel{\circ}{\nabla}_{\sharp \mathbf{v}^{*}} d f\right. \\
& Z)+\frac{1}{2}\left(\stackrel{\circ}{\nabla}_{Z} \mathbf{v}^{*}\right)(d f, d f), \\
\frac{1}{\operatorname{rank} \mathcal{H}}\left(\Delta_{\mathbf{h}}^{\prime} f\right)^{2} & \geq \frac{1}{n}\left(\Delta_{\mathbf{h}}^{\prime} f+Z f\right)^{2}-\frac{1}{n-\operatorname{rank} \mathcal{H}}(Z f)^{2}
\end{aligned}
$$

which hold for any vector field $Z$ (not necessarily vertical).

The proof of Proposition 3.13 also shows why it is complicated to extend this formalism to the more general case. If $\operatorname{pr}_{\mathcal{H}} Z \neq 0$, then the term $\ell d f\left(\nabla_{\sharp \mathbf{h}^{*}} d f \operatorname{pr}_{\mathcal{H}} Z\right)$ requires a lower bound on the form $\ell b \Gamma^{\mathbf{h}^{*}}(f)+\ell b \Gamma^{\mathbf{v}^{*}}(f)$ or $b \Gamma^{\mathbf{h}^{*}}(f)+\ell^{2} b \Gamma \mathbf{v}^{*}(f)$, both of which would be outside of our formalism.

3.8. Generalization to the case when $\mathcal{V}$ is not integrable. Not every vector bundle has an integrable complement [11, not to mention a metric-preserving one. We give a brief comment on how our results can be generalized to the case when $\mathcal{V}$ is not integrable.

Let $\mathcal{R}$ be defined as in (3.2) and let $\overline{\mathcal{R}}$ be defined by

$$
\overline{\mathcal{R}}(A, Z):=\operatorname{pr}_{\mathcal{H}}\left[\operatorname{pr}_{\mathcal{V}} A, \operatorname{pr}_{\mathcal{V}} Z\right], \quad A, Z \in \Gamma(T M) .
$$

We will adopt the terminology of [17, Ch II.8] and call $\mathcal{R}$ and $\overline{\mathcal{R}}$ respectively the curvature and the co-curvature of $\mathcal{H}$. Then our theory can still be applied with the following modifications. 
(a) We consider a complement $\mathcal{V}$ as metric-preserving if

$$
\operatorname{pr}_{\mathcal{H}}^{*} \mathcal{L}_{V}\left(\operatorname{pr}_{\mathcal{H}}^{*} \mathbf{h}\right)=0, \quad V \in \Gamma(\mathcal{V})
$$

Notice the difference between the formula above and (3.1). In fact, (3.1) holds if and only if (3.10) holds and $\mathcal{V}$ is integrable. Note that $(3.10)$ is equivalent to stating that $\stackrel{\circ}{\nabla}^{*}=0$ with respect to any connection $\nabla^{\circ}$ defined as in (2.3) using some metric $\mathbf{g}$ which tames $\mathbf{h}$ and makes $\mathcal{V}$ the orthogonal complement of $\mathcal{H}$.

(b) In Section 3, we now have that $T^{\circ}=-\mathcal{R}-\overline{\mathcal{R}}$ in Lemma 3.2 (c). As a consequence, in Lemma 3.3 (c), we obtain

$$
\mathbf{g}\left(R^{\stackrel{\circ}{\nabla}}(A, Y) Z-R^{\stackrel{\circ}{\nabla}}(A, Z) Y, A\right)=\mathbf{g}(\overline{\mathcal{R}}(Y, \mathcal{R}(Z, A))-\overline{\mathcal{R}}(Z, \mathcal{R}(Y, A)), A) .
$$

Hence,

$$
\mathbf{g}\left(R^{\stackrel{\circ}{\nabla}}\left(A, \operatorname{pr}_{\mathcal{H}} Y\right) \operatorname{pr}_{\mathcal{H}} Z, A\right)=\mathbf{g}\left(R^{\stackrel{\circ}{\nabla}}\left(A, \operatorname{pr}_{\mathcal{H}} Z\right) \operatorname{pr}_{\mathcal{H}} Y, A\right)
$$

however, we now have $\mathbf{g}\left(R^{\triangleright}\left(A, \operatorname{pr}_{\mathcal{V}} Y\right) Z, A\right)=\mathbf{g}(\overline{\mathcal{R}}(Y, \mathcal{R}(Z, A)), A)$.

(c) In Section 3.4 Lemma 3.11 still holds, but since $\nabla^{\circ}-\stackrel{\infty}{\nabla}$ is different, in Corollary 3.11 we have

$$
\left.\stackrel{\circ}{\nabla}_{Z} V_{s}\right|_{x_{0}}=-\left.\frac{1}{2} \sharp\left(\stackrel{\circ}{\nabla}_{Z} \mathbf{g}\right)\left(V_{s}, \cdot\right)\right|_{x_{0}}+\left.\frac{1}{2} \sharp \mathbf{g}\left(Z, \overline{\mathcal{R}}\left(V_{s}, \cdot\right)\right)\right|_{x_{0}} .
$$

(d) In the proof of Theorem 3.5 in Section 3.5 , the only difference is that in Eq. (3.7), we cannot be sure that the term

$$
\sum_{i=1}^{n} \mathbf{g}\left(R^{\stackrel{\nabla}{\nabla}}\left(A_{i}, \sharp^{\mathbf{v}^{*}} d f\right) \sharp^{\mathbf{h}^{*}} d f, A_{i}\right)=\operatorname{tr} \overline{\mathcal{R}}\left(\sharp^{\mathbf{v}^{*}} d f, \mathcal{R}\left(\sharp^{\mathbf{h}^{*}} d f, \cdot\right)\right)
$$

vanishes. Hence, we require the separate assumption that for any vector $v \in$ $T M$ on $M$, we have

$$
\operatorname{tr} \overline{\mathcal{R}}(v, \mathcal{R}(v, \cdot))=0 .
$$

The same assumption also guarantees that

$$
\operatorname{tr} R^{\stackrel{\circ}{\nabla}}\left(\operatorname{pr}_{\mathcal{H}} \cdot, Z_{1}\right) Z_{2}=\operatorname{tr} R^{\circ}\left(\operatorname{pr}_{\mathcal{H}} \cdot, \operatorname{pr}_{\mathcal{H}} Z_{1}\right) \operatorname{pr}_{\mathcal{H}} Z_{2},
$$

so the definition of $\operatorname{Ric}_{\mathcal{H}}$ in Proposition 3.4 is still valid. If this does indeed hold, then Theorem 3.5 remains true even if $\mathcal{V}$ is not integrable. As a consequence, all further results in this paper and in Part II also hold in this case. Since we do not have any geometric interpretation for the requirement (3.11), we prefer to mainly consider the case when $\mathcal{V}$ is integrable.

The only exception are the results in Section 3.7 Even if (3.11) is satisfied, these results do do not hold when $\mathcal{V}$ is not integrable.

Example 3.14. Consider the Lie algebra $\mathfrak{s u}(2)$ with basis $A, B, C$ satisfying commutation relations

$$
[A, B]=C, \quad[A, C]=-B, \quad[B, C]=C .
$$

Consider its complexification, which is isomorphic to $\mathfrak{s l}(2, \mathbb{C})$. Define a sub-Riemannian manifold $(\mathrm{SL}(2, \mathbb{C}), \mathcal{H}, \mathbf{h})$ by considering $i A, i B, i C$ and $C$ as an orthonormal basis for $\mathcal{H}$. Here, we have used the same symbol for an element of the Lie algebra and its corresponding left invariant vector field. Then $\mathcal{V}$ spanned by $A$ and $B$ is a metric-preserving complement that is not integrable, but satisfies (3.11). 


\section{SPECTRAL GAP AND EXAMPLES}

4.1. The curvature-dimension inequality and a bound for the spectral gap. Let $(M, \mathcal{H}, \mathbf{h})$ be a compact sub-Riemannian manifold where $\mathcal{H}$ is bracket-generating. Let $L$ be a smooth second order operator without constant term satisfying $\mathbf{q}_{L}=\mathbf{h}^{*}$. Assume also that $L$ is symmetric with respect to some volume form vol on $M$. Since the metric $\mathrm{d}_{c c}$ induced by $\mathbf{h}^{*}$ is obviously complete on $M$, we have that $L$ is essentially self-adjoint on $C_{c}^{\infty}(M)$ by [24, Sec 12]. Denote its (unique) self-adjoint extension to an operator on $L^{2}(M$, vol $)$ also by $L$.

Proposition 4.1. Assume that $L$ satisfies (CD*) with $\rho_{2,0}>0$. Let $\lambda$ be any nonzero eigenvalue of $L$. Then

$$
\frac{n \rho_{2,0}}{n+\rho_{2,0}(n-1)}\left(\rho_{1}-\frac{k_{2}}{\rho_{2,0}}\right) \leq-\lambda, \quad k_{2}=\max \left\{0,-\rho_{2,1}\right\} .
$$

Proof. Since $\mathcal{H}$ is bracket-generating, we know that $L-\lambda$ is hypoelliptic for any $\lambda$ by [15, so all eigenfunctions of $L$ are smooth. If we write $\langle f, g\rangle=\int_{M} f g d$ vol, note that $\int_{M} L f d \mathrm{vol}=\langle L f, 1\rangle=0$ and $\int_{M} \Gamma^{\mathbf{h}^{*}}(f, g) d \mathrm{vol}=-\langle f, L g\rangle$ for $f, g \in C^{\infty}(M)$. Since $L$ is a nonpositive operator, any nonzero eigenvalue is negative. From (CD*) we get

$$
\begin{aligned}
\int_{M}\left(\Gamma_{2}^{\mathrm{h}^{*}}(f)+\ell \Gamma_{2}^{\mathrm{v}^{*}}(f)\right) d \mathrm{vol}=\langle L f, L f\rangle-\left\langle\Gamma^{\mathrm{v}^{*}}(f, L f), \ell\right\rangle \\
\quad \geq \frac{1}{n}\langle L f, L f\rangle-\left(\rho_{1}-\frac{1}{\ell}\right)\langle f, L f\rangle+\left\langle\Gamma^{\mathrm{v}^{*}}(f), \rho_{2,0}+\ell \rho_{2,1}\right\rangle .
\end{aligned}
$$

Hence, if $f$ satisfies $L f=\lambda f$, then

$$
\frac{n-1}{n} \lambda^{2}\langle f, f\rangle \geq-\lambda\left(\rho_{1}-\frac{1}{\ell}\right)\langle f, f\rangle+\left\langle\Gamma^{\mathbf{v}^{*}}(f), \rho_{2,0}+\ell\left(\rho_{2,1}+\lambda\right)\right\rangle .
$$

We choose $\ell=\frac{\rho_{2,0}}{-\lambda+k_{2}}$ and obtain

$$
\frac{n-1}{n} \lambda^{2} \geq-\lambda\left(\rho_{1}-\frac{-\lambda+k_{2}}{\rho_{2,0}}\right)
$$

from which the result follows.

Let $\mathbf{g}$ be a Riemannian metric taming $\mathbf{h}$ such that the orthogonal complement $\mathcal{V}$ of $\mathcal{H}$ is integrable. We consider the special case when $\stackrel{\circ}{\nabla} \mathbf{g}=0$ where $\stackrel{\circ}{\nabla}^{\circ}$ is defined as in (2.3). Then $\mathcal{V}$ is a metric-preserving complement and $\nabla \mathbf{v}^{*}=0$ where $\left.\mathbf{g}\right|_{\mathcal{V}}=\mathbf{v}$. Let vol be the volume form of $\mathbf{g}$ and let $\Delta_{\mathbf{h}}$ the sub-Laplacian of vol, which will also be the sub-Laplacian of $\mathcal{V}$.

Corollary 4.2. Assume that the assumptions of Theorem 3.5 hold with

$$
\kappa=\frac{1}{2} \rho_{\mathcal{H}} m_{\mathcal{R}}^{2}-\mathscr{M}_{\mathcal{H} \mathcal{V}}^{2}>0 .
$$

Then, for any $\operatorname{rank} \mathcal{H} \leq n \leq \infty$,

$$
\left(\frac{2 \kappa}{2 \mathscr{M}_{\mathcal{H} \mathcal{V}}+m_{\mathcal{R}} \sqrt{2 \rho_{\mathcal{H}}+2 \frac{n-1}{n} \kappa}}\right)^{2} \leq-\lambda .
$$

Proof. This follows from formulas (3.4) and by choosing the optimal value of $c$. 
4.2. Privileged metrics. Let $(M, \mathcal{H}, \mathbf{h})$ be a sub-Riemannian manifold with $\mathcal{H}$ bracket-generating and equiregular of step $r$ as in Remark 2.2. Let $\operatorname{dim} M=n+\nu$ with $n$ being the rank of $\mathcal{H}$. Any Riemannian metric $\widetilde{\mathbf{g}}$ on $M$ such that $\left.\widetilde{\mathbf{g}}\right|_{\mathcal{H}}=\mathbf{h}$, gives us automatically a splitting

$$
T M=\mathcal{H} \oplus \mathcal{V}^{1} \oplus \cdots \oplus \mathcal{V}^{r-1}
$$

where $\mathcal{V}^{k}$ is the orthogonal complement of $\mathcal{H}^{k}$ in $\mathcal{H}^{k+1}$. Conversely, associated to each such splitting, there exist a canonical way of constructing a Riemannian metric $\widetilde{\mathbf{g}}$ taming $\mathbf{h}$, which we will call privileged. Define a vector bundle morphism

$$
\Psi: \mathcal{H} \oplus \mathcal{H}^{\otimes 2} \oplus \cdots \oplus \mathcal{H}^{\otimes r} \rightarrow \mathcal{H} \oplus \mathcal{V}^{1} \oplus \cdots \oplus \mathcal{V}^{r-1},
$$

such that $\Psi$ is the identity on the first component, while elements in $\mathcal{H}^{\otimes j}, j \geq 2$ are sent to $\mathcal{V}^{j-1}$ by

$$
A_{1} \otimes A_{2} \otimes \cdots \otimes A_{j} \mapsto \operatorname{pr}_{\mathcal{V}_{j-1}}\left[A_{1},\left[A_{2}\left[\cdots\left[A_{j-1}, A_{j}\right]\right] \cdots\right]\right] .
$$

Giving $\mathcal{H}^{\otimes j}$ the metric $\mathbf{h}^{\otimes j}, \Psi$ induces a metric $\widetilde{\mathbf{g}}$ on $T M$ by requiring that $\left.\Psi\right|_{(\operatorname{ker} \Phi)^{\perp}}$ is a fiberwise isometry.

Assume that $\mathcal{V}=\oplus_{k=1}^{r-1} \mathcal{V}_{k}$ is an integrable metric-preserving complement. Define $b$ as the minimal number such that $\|\mathcal{R}(v, .)\|_{\widetilde{\mathbf{g}}^{*} \otimes \widetilde{\mathbf{g}}} \leq b\left\|\operatorname{pr}_{\mathcal{H}} v\right\|_{\widetilde{\mathbf{g}}}$ for any $v \in T M$. Note that

$$
\frac{2 \operatorname{dim} \mathcal{V}_{1}}{n}=\frac{2}{n}\|\mathcal{R}\|_{\wedge^{2} \widetilde{\mathbf{g}} \otimes \mathbf{g}}^{2} \leq b^{2} \leq 2\|\mathcal{R}\|_{\wedge 2 \widetilde{\mathbf{g}} \otimes \mathbf{g}}^{2}=2 \operatorname{dim} \mathcal{V}_{1}
$$

We normalize the vertical part of the metric by defining

$$
\mathbf{v}=\frac{1}{b^{2}} \widetilde{\mathbf{g}} \mid \mathcal{V} \quad \text { and } \mathbf{g}=\operatorname{pr}_{\mathcal{H}}^{*} \mathbf{h}+\operatorname{pr}_{\mathcal{V}}^{*} \mathbf{v}
$$

Then $\mathscr{M}_{\mathcal{R}}=1$, while $m_{\mathcal{R}}=\frac{1}{b}$ if $r=2$ and 0 otherwise. Furthermore, if $\mathcal{H}$ is of step 2 and $\stackrel{\circ}{\nabla}_{A} \mathcal{R}=0$ for any $A \in \Gamma(\mathcal{H})$, then $\stackrel{\circ}{\nabla}^{*}=0$ and Ric $\mathcal{H V}=0$. Hence, for this special case, the sub-Laplacian $\Delta_{\mathbf{h}}$ of $\mathcal{V}$ or equivalently the volume form of $\mathbf{g}$, satisfies curvature-dimension inequality

$$
\Gamma_{2}^{\mathbf{h}^{*}+\ell \mathbf{v}^{*}}(f) \geq \frac{1}{n}\left(\Delta_{\mathbf{h}} f\right)^{2}+\left(\rho_{\mathcal{H}}-\ell^{-1}\right) \Gamma^{\mathbf{h}^{*}}(f)+\frac{1}{2 b^{2}} \Gamma^{\mathbf{v}^{*}}(f),
$$

for any $\ell>0$. As a consequence, if $M$ is compact with $\rho_{\mathcal{H}}>0$ and $\lambda$ is a non-zero eigenvalue of $\Delta_{\mathbf{h}}$ then

from Proposition 4.1 .

$$
\frac{n}{n\left(2 b^{2}+1\right)-1} \rho_{\mathcal{H}} \leq-\lambda
$$

The volume forms of all privileged metric taming $\mathbf{h}$ coincide and is called Popp's measure. For more details, see [2].

4.3. Sub-Riemannian manifolds with transverse symmetries. For two Riemann manifolds $\left(M_{j}, \mathcal{H}_{j}, \mathbf{h}_{j}\right), j=1,2$, a sub-Riemannian isometry $\phi: M_{1} \rightarrow M_{2}$ is a diffeomorphism such that $\phi^{*} \mathbf{h}_{2}^{*}=\mathbf{h}_{1}^{*}$. The later requirement can equivalently be written as $\phi_{*} \mathcal{H}_{1} \subseteq \mathcal{H}_{2}$ and $\mathbf{h}_{2}\left(\phi_{*} v, \phi_{*} v\right)=\mathbf{h}_{1}(v, v)$ for any $v \in \mathcal{H}_{1}$. An infinitesimal isometry of a sub-Riemannian manifold $(M, \mathcal{H}, \mathbf{h})$ is a vector field $V$ such that

$$
\mathcal{L}_{V} \mathbf{h}^{*}=0 .
$$

If $V$ is complete with flow $\phi_{t}$, then this flow is an isometry from $M$ to itself for every fixed $t$. 
We will introduce sub-Riemannian manifolds with transverse symmetries according to the definition found in $[8$. This is a special case of a sub-Riemannian manifold with a metric-preserving complement and consists of sub-Riemannian manifolds $(M, \mathcal{H}, \mathbf{h})$ with an integrable complement $\mathcal{V}$ spanned by $\nu$ linearly independent vector fields $V_{1}, \ldots, V_{\nu}$, such that each of these vector fields is an infinitesimal isometry. The subbundle $\mathcal{V}$ will then be a metric preserving complement. If $\mathbf{v}$ is the metric on $\mathcal{V}$ defined such that $V_{1}, \ldots, V_{\nu}$ forms an orthonormal basis, then $\stackrel{\circ}{\nabla}^{*}=0$. Hence, a complement spanned by transverse symmetries gives us a totally geodesic foliation.

Since we assume that $\mathcal{H}$ was bracket-generating, it follows that the span $V_{1}, \ldots, V_{\nu}$ actually is a subalgebra of $\Gamma(T M)$. Indeed, since $\mathcal{H}$ is bracket-generating, any function $f \in C^{\infty}(M)$ satisfying $A f=0$ for all $A \in \Gamma(\mathcal{H})$ must be a constant. Since $\left[V_{i}, V_{j}\right]=\sum_{s=1}^{\nu} f_{s} V_{s}$ must also be an infinitesimal isometry for any $i, j$, we have that for all $A \in \Gamma(\mathcal{H})$,

$$
0=\operatorname{pr}_{\mathcal{V}}\left[A,\left[V_{i}, V_{j}\right]\right]=\sum_{s=1}^{\nu}\left(A f_{s}\right) V_{s} .
$$

It follows that each $f_{s}$ is constant, and the span of $V_{1}, \ldots, V_{\nu}$ is a subalgebra. If all of the vector fields are complete, we get a corresponding group action. We will then be in the following case.

Example 4.3. Let $G$ be a Lie group with Lie algebra $\mathfrak{g}$. Consider a principal bundle $G \rightarrow M \stackrel{\pi}{\rightarrow} B$ over a Riemannian manifold $(B, \breve{\mathbf{g}})$ with $G$ acting on the right. An Ehresmann connection $\mathcal{H}$ on $\pi$ is called principal if $\mathcal{H}_{x} \cdot a=\mathcal{H}_{x \cdot a}$. For any such principal connection $\mathcal{H}$, define $\mathbf{h}=\left.\pi^{*} \mathbf{\mathbf { g }}\right|_{\mathcal{H}}$. Then $G$ acts on $(M, \mathcal{H}, \mathbf{h})$ by isometries and so, for each $A \in \mathfrak{g}$, the vector field $\sigma(A)$ defined by

$$
\left.\sigma(A)\right|_{x}=\left.\frac{d}{d t}\right|_{t=0} x \cdot \exp _{G}(A t)
$$

is an infinitesimal isometry. This is hence a sub-Riemannian manifold with transverse symmetries. Let $\omega: T M \rightarrow \mathfrak{g}$ be principal curvature form of $\mathcal{H}$, i.e. the $\mathfrak{g}$-valued one-form defined by

$$
\text { ker } \omega=\mathcal{H}, \quad \omega(\sigma(A))=A .
$$

Then for any inner product $\langle\cdot, \cdot \cdot\rangle$ on $\mathfrak{g}$, define a Riemannian metric $\mathbf{g}$ by $\mathbf{g}(v, v)=$ $\check{\mathbf{g}}\left(\pi_{*} v, \pi_{*} v\right)+\langle\omega(v), \omega(v)\rangle, v \in T M$. This Riemannian metric satisfies $\nabla^{\circ} \mathbf{g}=0$. We assume that the vertical part of $\mathbf{g}$ is normalized so that $\mathscr{M}_{\mathcal{R}}=1$.

In general, the metric $\mathbf{g}$ is not invariant under the group action. The latter only hold if $\langle., . \cdot\rangle$ is bi-invariant inner product on $\mathfrak{g}$. If such an inner product exist, it induces a metric tensor $\breve{\mathbf{v}}$ on the vector bundle $\operatorname{Ad}(M) \rightarrow B$, where $\operatorname{Ad}(M)$ is the quotient of $M \times \mathfrak{g}$ by the action $(x, A) \cdot a=\left(x \cdot a, \operatorname{Ad}\left(a^{-1}\right) A\right)$. Any $\mathfrak{g}$-valued from $\alpha$ on $M$ that vanish on $\mathcal{V}$ and satisfies $\alpha\left(Z_{1} \cdot a, \ldots, Z_{j} \cdot a\right)=\operatorname{Ad}\left(a^{-1}\right) \alpha\left(Z_{1}, \ldots, Z_{j}\right)$ can be considered as an $\operatorname{Ad}(M)$-valued form on $B$. This includes the curvature form $\Omega\left(Z_{1}, Z_{2}\right)=d \omega\left(Z_{1}, Z_{2}\right)+\left[\omega\left(Z_{1}\right), \omega\left(Z_{2}\right)\right]=-\omega\left(\mathcal{R}\left(Z_{1}, Z_{2}\right)\right)$.

Conversely, any section $F$ of $\operatorname{Ad}(M)$ can be considered a function $F: M \rightarrow \mathfrak{g}$ satisfying $F(x \cdot a)=\operatorname{Ad}\left(a^{-1}\right) F(x)$. Define a connection $\nabla^{\omega}$ on $\operatorname{Ad}(M)$ by formula $\nabla_{\check{Z}}^{\omega} F=d F(h \check{Z})$ for $\check{Z} \in \Gamma(T B)$ and let $d_{\nabla} \omega$ be the corresponding covariant exterior derivative of $\operatorname{Ad}(M)$-valued forms on $B$. If we consider $\Omega$ as a $\operatorname{Ad}(M)$-valued 2-form and $\delta_{\nabla^{\omega}}$ as the formal dual of $d_{\nabla^{\omega}}$, then

$$
\mathscr{M}_{\mathcal{H V}}=\sup _{B}\left\|\delta_{\nabla^{\omega}} \Omega\right\|_{\check{\mathbf{g}} \otimes \check{\mathbf{v}}}
$$


In particular, $\operatorname{Ric} \mathcal{H \mathcal { V }}=0$ if and only if $\delta_{\nabla^{\omega}} \Omega=0$, which is the definition of a Yang-Mills connection on $\pi$.

4.4. Invariant sub-Riemannian structures on Lie groups. Let $G$ be a Lie group with Lie algebra $\mathfrak{g}$. Let $G$ have dimension $n+\nu$. Choose a subspace $\mathfrak{h} \subseteq \mathfrak{g}$ of dimension $n$ which generate the entire Lie algebra, and give this subspace an inner product. Define a vector bundle $\mathcal{H}$ by left translation of $\mathfrak{h}$. Use the inner product on $\mathfrak{h}$ to induce a left invariant metric $\mathbf{h}$ on $\mathcal{H}$. This gives us a sub-Riemannian manifold $(G, \mathcal{H}, \mathbf{h})$ with a left-invariant structure, i.e. $G$ acts on the left by isometries. This means that right invariant vector fields are infinitesimal isometries, however, we cannot be sure that we have a complement spanned by such vector fields. This is the case if and only if there exist a subspace $\mathfrak{k}$ of $\mathfrak{g}$ such that $\operatorname{Ad}(a) \mathfrak{k}$ is a complement of $\mathfrak{h}$ for any $a \in G$. Consider the special case when $\mathfrak{k}$ is a subalgebra of $\mathfrak{g}$ with corresponding subgroup $K$ and $\mathfrak{g}=\mathfrak{h} \oplus \mathfrak{k}$ as a vector space.

(a) Define $\mathcal{V}^{l}$ by left translation of $\mathfrak{k}$. Then $\mathcal{V}$ is a complement to $\mathcal{H}$, but this is not in general spanned by infinitesimal isometries. If $K$ is closed, $\mathcal{H}$ can be considered as an Ehresmann connection on $\pi: G \rightarrow G / K$, but it is not principal in general and we cannot necessarily consider the metric $\mathbf{h}$ as lifted from $G / K$.

(b) Define $\mathcal{V}^{r}$ by right translation of $\mathfrak{k}$. Then $\mathcal{V}^{r}$ is spanned by infinitesimal isometries. It is a complement if and only if $\operatorname{Ad}(a) \mathfrak{k}$ is a complement to $\mathfrak{h}$ for every $a \in G$. If the latter holds and $K$ is closed, $\mathcal{H}$ can be considered as an Ehresmann connection on $\pi: G \rightarrow K \backslash G$.

(c) If $\mathfrak{k}$ is an ideal (and $K$ a normal subgroup as a result) then $\mathcal{V}^{l}=\mathcal{V}^{r}$ is a complement spanned by infinitesimal isometries.

Example 4.4 (Free step-2 nilpotent Lie groups). Let $\mathfrak{h}$ be an inner product space of dimension $n$ and define $\mathfrak{k}=\bigwedge^{2} \mathfrak{h}$ with the inner product induced by the product on $\mathfrak{h}$. Define a Lie algebra $\mathfrak{g}$ as the vector space $\mathfrak{g}=\mathfrak{h} \oplus \mathfrak{k}$ with brackets [.,. .] such that $\mathfrak{k}$ is the center and for any $A, B \in \mathfrak{h}$,

$$
[A, B]=A \wedge B \in \mathfrak{k}
$$

Then $\mathfrak{g}$ is a step 2 nilpotent Lie algebra of dimension $n(n+1) / 2$. Using the inner products on $\mathfrak{h}$ and $\mathfrak{k}$ and defining these two spaces as orthogonal, we get an inner product on $\mathfrak{g}$.

Let $G$ be a simply connected Lie group with Lie algebra $\mathfrak{g}$ and normal subgroup $K$ corresponding to $\mathfrak{k}$. Define $\mathcal{H}$ and $\mathcal{V}$ by left translation of respectively $\mathfrak{h}$ and $\mathfrak{k}$. Give $G$ a Riemannian metric by left translation of the inner product on $\mathfrak{g}$. If we consider the inner product space $\mathfrak{h}$ as a flat Riemannian manifold, then

$$
\pi: G \rightarrow G / K \cong \mathfrak{h},
$$

is a Riemannian submersion with ker $\pi_{*}=\mathcal{V}$ and with $\mathcal{H}$ as an Ehresmann connection. Also $\widetilde{\mathrm{g}}$ coincides with the privileged metric of Section 4.2 .

Since

$$
\|\mathcal{R}(v, \cdot)\|_{\widetilde{\mathbf{g}}^{*} \otimes \widetilde{\mathbf{g}}}^{2}=(n-1)\left\|\operatorname{pr}_{\mathcal{H}} v\right\|_{\widetilde{\mathbf{g}}}^{2}, \quad v \in T M
$$

we normalize the vertical part by defining

$$
\mathbf{g}=\left.\operatorname{pr}_{\mathcal{H}}^{*} \widetilde{\mathbf{g}}\right|_{\mathcal{H}}+\left.\frac{1}{n-1} \operatorname{pr}_{\mathcal{V}}^{*} \widetilde{\mathbf{g}}\right|_{\mathcal{V}}
$$


With respect to this normalized metric,

$$
\mathscr{M}_{\mathcal{R}}=1, \quad m_{\mathcal{R}}=\frac{1}{\sqrt{n-1}}, \quad \stackrel{\circ}{\nabla} \mathbf{g}=0, \quad \mathscr{M}_{\mathcal{H V}}=0 .
$$

and $\rho_{\mathcal{H}}=0$ since $\mathfrak{h}$ is flat. Defining $\Delta_{\mathbf{h}}$ as the sub-Laplacian of $\mathcal{V}$ or equivalently the volume form of $\mathbf{g}$, we obtain inequality

$$
\Gamma_{2}^{\mathbf{h}^{*}+\ell \mathbf{v}^{*}}(f) \geq \frac{1}{n}\left(\Delta_{\mathbf{h}} f\right)^{2}-\frac{1}{\ell} \Gamma^{\mathbf{h}^{*}}(f)+\frac{1}{2(n-1)} \Gamma^{\mathbf{v}^{*}}(f) .
$$

Remark 4.5. Let $\mathbf{g}$ be a left invariant metric on the Lie group $G$, with $\mathbf{h}$ and $\mathbf{v}$ as the respective restrictions of $\mathbf{g}$ to a left invariant subbundle $\mathcal{H}$ and its orthogonal complement $\mathcal{V}$. If $\mathcal{V}$ is a metric-preserving complement of $(G, \mathcal{H}, \mathbf{h})$, the conditions of Theorem 3.5 hold, but we do not necessarily have $\stackrel{\circ}{\nabla} \mathrm{g}=0$.

4.5. Sub-Riemannian manifolds with several metric-preserving complements. The choice of metric preserving complement may not be unique and give different results in general. We give two examples of this.

Example 4.6. Let $\mathfrak{g}$ be a compact semi-simple Lie algebra of dimension $n$. The term "compact Lie algebra" is here used to mean that the Killing form

$$
(A, B) \mapsto \operatorname{tr} \operatorname{ad}(A) \operatorname{ad}(B)
$$

is negative definite. We remark that when $\mathfrak{g}$ is semi-simple, then $[\mathfrak{g}, \mathfrak{g}]=\mathfrak{g}$. Define and inner product

$$
\langle A, B\rangle=-\frac{1}{4 \rho} \operatorname{tr} \operatorname{ad}(A) \operatorname{ad}(B),
$$

for some $\rho>0$. Note that if we use this inner product to induce a product on $\operatorname{End}(\mathfrak{g}) \cong \mathfrak{g}^{*} \otimes \mathfrak{g}$, then $\|\operatorname{ad}(A)\|^{2}=4 \rho\|A\|^{2}$.

Let $G$ be a (compact) Lie group with with Lie algebra $\mathfrak{g}$ and let $\breve{\mathbf{g}}$ be the Riemannian metric on $G$ obtained by left (or right) translation of $\langle.,$.$\rangle . From standard$ theory of bi-invariant metrics on Lie groups, it follows that $\operatorname{Ric}_{\breve{\mathrm{g}}}(Z, Z)=\rho\|Z\|_{\mathrm{g}}^{2}$ pointwise for any vector field $Z$, so $G$ has Ricci lower bound $\rho>0$.

In what follows, we will always use the same symbol for an element in a Lie algebra and the corresponding left invariant vector field.

(a) Define

$$
\mathfrak{h}=\{(A, 2 A) \in \mathfrak{g} \oplus \mathfrak{g}: A \in \mathfrak{g}\} .
$$

From our assumptions, we know that $\mathfrak{h}+[\mathfrak{h}, \mathfrak{h}]=\mathfrak{g} \oplus \mathfrak{g}$. Define $\mathcal{H}$ by left translation on $G \times G$. Then $\mathcal{H}$ is an Ehresmann connections of the following submersions

$$
\pi^{j}: G \times G \rightarrow G, \quad \pi^{j}\left(a_{1}, a_{2}\right)=a_{j} \quad j=1,2,\left(a_{1}, a_{2}\right) \in G .
$$

Then the pullback of $\breve{\mathbf{g}}$ by $\pi^{1}$ or of $\frac{1}{4} \breve{\mathbf{g}}$ by $\pi^{2}$ gives us the same metric $\mathbf{h}$ when restricted to $\mathcal{H}$. We can write this as

$$
\mathbf{h}((A, 2 A),(A, 2 A))=\langle A, A\rangle, \quad A \in \mathfrak{g} .
$$

The sub-Laplacian defined relative to either $\operatorname{ker} \pi_{*}^{1}$ or $\operatorname{ker} \pi_{*}^{2}$ is

$$
\Delta_{\mathbf{h}}=\sum_{i=1}^{n}\left(A_{i}, 2 A_{i}\right)^{2}
$$


where $A_{1}, \ldots, A_{n}$ is some orthonormal basis of $\mathfrak{g}$. First consider, $\mathcal{V}_{1}=\operatorname{ker} \pi_{*}^{1}$ spanned by elements $(0, A), A \in \mathfrak{g}$, with the metric $\mathbf{v}_{1}$ given as

$$
\|(0, A)\|_{\mathbf{v}^{1}}^{2}=\frac{1}{16 \rho}\langle A, A\rangle .
$$

The constants we obtain are

$$
\begin{aligned}
& \mathcal{R}((A, 2 A),(B, 2 B))=2(0,[A, B]), \quad \mathscr{M}_{\nabla_{\mathbf{v}}^{*}}^{*}=0, \\
& \mathscr{M}_{\mathcal{R}}=1, \quad \rho_{\mathcal{H}}=\rho, \quad \mathscr{M}_{\mathcal{H} \mathcal{V}}=\frac{3}{8}, \quad m_{\mathcal{R}}=\frac{1}{2},
\end{aligned}
$$

giving us the inequality

$$
\Gamma_{2}^{\mathbf{h}^{*}+\ell \mathbf{v}_{1}^{*}}(f) \geq \frac{1}{n}\left(\Delta_{\mathbf{h}} f\right)^{2}+\left(\rho-c^{-1}-\ell^{-1}\right) \Gamma^{\mathbf{h}^{*}}(f)+\left(\frac{1}{8}-\frac{9 c}{64}\right) \Gamma^{\mathbf{v}_{1}^{*}}(f),
$$

for any $c>0$. However, by choosing $\mathcal{V}_{2}=\operatorname{ker} \pi_{*}^{2}$, with metric

$$
\|(A, 0)\|_{\mathbf{v}^{2}}^{2}=\frac{1}{4 \rho}\langle A, A\rangle .
$$

we obtain a better result

$$
\begin{gathered}
\mathcal{R}((A, 2 A),(B, 2 B))=-([A, B], 0), \quad \mathscr{M}_{\odot \mathbf{\nabla}_{2}^{*}}=0 \\
\mathscr{M}_{\mathcal{R}}=1, \quad \rho_{\mathcal{H}}=4 \rho, \quad \mathscr{M}_{\mathcal{H V}}=0, \quad m_{\mathcal{R}}=\frac{1}{\sqrt{2}}
\end{gathered}
$$

so that

$$
\Gamma_{2}^{\mathbf{h}^{*}+\ell \mathbf{v}_{2}^{*}}(f) \geq \frac{1}{n}\left(\Delta_{\mathbf{h}} f\right)^{2}+\left(4 \rho-\ell^{-1}\right) \Gamma^{\mathbf{h}^{*}}(f)+\frac{1}{4} \Gamma^{\mathbf{v}_{2}^{*}}(f) .
$$

From Proposition 4.1 and Eq. (4.3), we know that if $\lambda$ is any non-zero eigenvalue of $\Delta_{\mathbf{h}}$, then

$$
\frac{4 n}{5 n-1} \rho \leq-\lambda
$$

By contrast, we can not even obtain a spectral gap bound using inequality (4.2) unless $\rho>9 / 8$, and even then, the result from using $\mathbf{v}_{2}^{*}$ will give the better bound.

(b) Consider $\mathbb{R}^{n}$ as the trivial Lie algebra. Let $I: \mathfrak{g} \rightarrow \mathbb{R}^{n}$ be a bilinear map of vector spaces. Define $\mathfrak{h}$ as a subspace of $\mathfrak{g} \times \mathbb{R}^{n}$ by $(A, I(A)), A \in \mathfrak{g}$. Consider $\mathfrak{g} \times \mathbb{R}^{n}$ as the Lie algebra of $G \times \mathbb{R}^{n}$, where $\mathbb{R}^{n}$ is considered as a Lie group under + . Define $\mathcal{H}$ by left translation of $\mathfrak{h}$. This is an Ehresmann connection relative to both projections

$$
\pi^{1}: G \times \mathbb{R}^{n} \rightarrow G, \quad \pi^{2}: G \times \mathbb{R}^{n} \rightarrow \mathbb{R}^{n} .
$$

Give $G$ the metric $\breve{g}$ and give $\mathbb{R}^{n}$ a flat metric by the inner product $\langle I(\cdot), I(\cdot)\rangle$. Pulling back these metrics through respectively $\pi^{1}$ and $\pi^{2}$, we obtain the same sub-Riemannian metric $\mathbf{h}$ on $\mathcal{H}$ given by

$$
\|(A, I(A))\|_{\mathbf{h}}^{2}=\langle A, A\rangle,
$$

even though the geometry of $G$ and $\mathbb{R}^{n}$ are very different. The sub-Laplacians with respect to $\mathcal{V}^{1}=\operatorname{ker} \pi^{1}$ and $\mathcal{V}^{2}=\operatorname{ker} \pi^{2}$ also coincide; it is given by

$$
\Delta_{\mathbf{h}}=\sum_{i=1}^{n}(A, I(A))^{2}, \quad A_{1}, A_{2}, \ldots, A_{n} \text { an orthonormal basis of } \mathfrak{g} .
$$


We will leave out most of the calculations, and only state that if we define $\mathbf{v}_{j}$ on $\mathcal{V}^{j}=\operatorname{ker} \pi_{*}^{j}$ by

$$
\|(0, I(A))\|_{\mathbf{v}_{1}}^{2}=\frac{1}{4 \rho}\langle A, A\rangle, \quad\|(A, 0)\|_{\mathbf{v}_{2}}^{2}=\frac{1}{4 \rho}\langle A, A\rangle,
$$

then these metrics are appropriately normalized and we get inequalities

$$
\begin{aligned}
& \Gamma_{2}^{\mathbf{h}^{*}+\ell \mathbf{v}_{1}^{*}}(f) \geq \frac{1}{n}\left(\Delta_{\mathbf{h}} f\right)^{2}+\left(\rho-\frac{1}{c}-\frac{1}{\ell}\right) \Gamma^{\mathbf{h}^{*}}(f)+\left(\frac{1}{4}-c \rho\right) \Gamma^{\mathbf{v}_{1}^{*}}(f), \\
& \Gamma_{2}^{\mathbf{h}^{*}+\ell \mathbf{v}_{2}^{*}}(f) \geq \frac{1}{n}\left(\Delta_{\mathbf{h}} f\right)^{2}-\left(\frac{1}{c}+\frac{1}{\ell}\right) \Gamma^{\mathbf{h}^{*}}(f)+\left(\frac{1}{4}-c \rho\right) \Gamma^{\mathbf{v}_{2}^{*}}(f),
\end{aligned}
$$

that hold for any $c>0$ and $\ell>0$.

4.6. A non-integrable example. As usual, we use the same symbol for an element of the Lie algebra and the corresponding left invariant vector field. Consider the complexification of $\mathfrak{s u}(2)$ spanned over $\mathbb{C}$ by

$$
[A, B]=C, \quad[B, C]=A, \quad[C, A]=B .
$$

Consider $\mathfrak{s u}(2)^{\mathbb{C}}$ as the Lie algebra of $\mathrm{SL}(2, \mathbb{C})$ and on that Lie group, define (real) subbundles of the tangent bundle

$$
\mathcal{H}=\operatorname{span}\{i A, i B, i C, C\}, \quad \mathcal{V}=\operatorname{span}\{A, B\} .
$$

Define a Riemannian metric $\mathbf{g}$ such that $i A, i B, i C, C, \sqrt{2} A$ and $\sqrt{2} B$ forms an orthonormal basis. Define $\left.\mathbf{g}\right|_{\mathcal{H}}=\mathbf{h}$ and $\left.\mathbf{g}\right|_{\mathcal{V}}=\mathbf{v}$ and let $\Delta_{\mathbf{h}}^{\prime}$ be the sub-Laplacian of the sub-Riemannian manifold $(\mathcal{H}, \mathbf{h})$ with respect to the complement $\mathcal{V}$. It is simple to verify that

$$
\Delta_{\mathbf{h}}^{\prime}=(i A)^{2}+(i B)^{2}+(i C)^{2}+C^{2} .
$$

We caution the reader that $(i A)^{2}$ stands for the left invariant vector field corresponding the element $i A$ applied twice; it is in no way equal to $-A^{2}$.

Let $\mathcal{R}$ and $\overline{\mathcal{R}}$ be respectively the curvature and the co-curvature of $\mathcal{H}$. Note that

$$
\begin{aligned}
& \stackrel{\circ}{\nabla} \mathbf{h}^{*}=0, \quad \stackrel{\circ}{\nabla} \mathbf{v}^{*}=0, \\
& \operatorname{tr} \overline{\mathcal{R}}(v, \mathcal{R}(v, .))=0 \quad \text { for any } v \in T M, \\
& \operatorname{Ric}_{\mathcal{H}}(Y, Y)=-\frac{5}{2} \mathbf{g}(i A, Y)^{2}-\frac{5}{2} \mathbf{g}(i B, Y)^{2}-2 \mathbf{g}(i C, Y)^{2}+\frac{1}{2} \mathbf{g}(C, Y)^{2}, \\
& \operatorname{Ric}_{\mathcal{H} \mathcal{V}}=0, \quad \mathscr{M}_{\mathcal{R}}=1, \quad m_{\mathcal{R}}=1 .
\end{aligned}
$$

It follows that $\Delta_{\mathbf{h}}^{\prime}$ is also the sub-Laplacian of the volume form of $\mathbf{g}$ with curvaturedimension inequality

$$
\Gamma_{2}^{\mathbf{h}^{*}+\lambda \mathbf{v}^{*}}(f) \geq \frac{1}{4}\left(\Delta_{\mathbf{h}}^{\prime} f\right)^{2}-\left(\frac{5}{2}+\frac{1}{\lambda}\right) \Gamma^{\mathbf{h}^{*}}(f)+\frac{1}{2} \Gamma^{\mathbf{v}^{*}}(f) .
$$

\section{Summary of PART II}

We include a section here to illustrate what further results can be obtained from our curvature-dimension inequality $\mathrm{CD}^{*}$ of Theorem 3.5

Let $L$ be a second order operator. Let $X(x)$ be an $L$-diffusion with $X(x)=x$ and maximal lifetime $\tau(x)$. For bounded functions $f$, define $P_{t} f$ by

$$
P_{t} f(x)=\mathbb{E}\left[f\left(X_{t}(x)\right) 1_{t \leq \tau}\right] .
$$


Assume that $L$ satisfies an inequality $\left(\mathrm{CD}^{*}\right.$ with respect to some $\mathbf{v}^{*} \in \Gamma\left(\mathrm{Sym}^{2} T M\right)$. Let $C_{b}^{\infty}(M)$ denote the space of all smooth, bounded functions. We will introduce two important conditions.

(A) $P_{t} 1=1$ and for any $f \in C_{b}^{\infty}(M)$ with $\Gamma^{\mathbf{h}^{*}+\mathbf{v}^{*}}(f) \in C_{b}^{\infty}(M)$ and for every $T>0$, we have

$$
\sup _{t \in[0, T]}\left\|\Gamma^{\mathbf{h}^{*}+\mathbf{v}^{*}}\left(P_{t} f\right)\right\|_{L^{\infty}}<\infty
$$

(B) For any $f \in C^{\infty}(M)$, we have $\Gamma^{\mathbf{h}^{*}}\left(f, \Gamma^{\mathbf{v}^{*}}(f)\right)=\Gamma^{\mathbf{v}^{*}}\left(f, \Gamma^{\mathbf{h}^{*}}(f)\right)$.

We have the following concrete classes of sub-Riemannian manifolds satisfying these conditions.

Theorem 5.1 (Part II, Proposition 3.3, Theorem 3.4). (a) Assume that $\pi: M \rightarrow$ $B$ is a fiber-bundle with compact fibers over a Riemannian manifold $(B, \breve{\mathbf{g}})$. Let $\mathcal{H}$ be an Ehresmann connection on $\pi$ and define $\mathbf{h}=\left.\pi^{*} \breve{\mathbf{g}}\right|_{\mathcal{H}}$. Assume that the metric $\mathrm{d}_{c c}$ of $(M, \mathcal{H}, \mathbf{h})$ is complete and that the sub-Laplacian $\Delta_{\mathbf{h}}^{\prime}$ of $\mathcal{V}=\operatorname{ker} \pi_{*}$ satisfies (CD*). Then condition (A) holds.

(b) Let $M$ be a sub-Riemannian manifolds with an integrable, metric preserving complement $\mathcal{V}$. Assume that there exist a choice of $\mathbf{v}$ on $\mathcal{V}$ such that $\mathbf{g}=$ $\operatorname{pr}_{\mathcal{H}}^{*} \mathbf{h}+\operatorname{pr}_{\mathcal{V}}^{*} \mathbf{v}$ is a complete Riemannian metric and such that $\nabla^{\circ} \mathbf{v}^{*}=0$. Then the sub-Laplacian $\Delta_{\mathbf{h}}$ of $\mathcal{V}$ and the volume form of vol of $\mathbf{g}$ coincide. Assume that $\Delta_{\mathbf{h}}$ satisfies the assumptions of Theorem 3.5. Finally, assume that $m_{\mathcal{R}}>$ 0 . Then conditions $(\mathrm{A})$ and $(\mathrm{B})$ hold.

The result of Theorem 5.1 (b) is also valid for non-integrable complements of the type described in Section [3.8. For the special case when $\mathbf{h}^{*}$ is a complete Riemannian metric, $\mathbf{v}^{*}=0$ and $L$ is the Laplacian, (A) always holds when the curvature-dimension inequality holds. For this reason, we expect that the condition (A) will hold in more cases than the ones listed here.

Combined with our generalized curvature-dimension inequality, we have the following identities.

Theorem 5.2. (Part II, Proposition 3.8) Assume that L satisfies (CD*) with respect to $\mathbf{v}^{*}$ and that (A) holds. Assume also that $L$ is symmetric with respect to the volume form vol, i.e. $\int_{M} f L g d \mathrm{vol}=\int_{M} g L f d \mathrm{vol}$ for any $f, g \in C_{c}^{\infty}(M)$. Finally, assume that $\mathbf{g}^{*}=\mathbf{h}^{*}+\mathbf{v}^{*}$ is the co-metric of a complete Riemannian metric.

(a) If $\rho_{1} \geq \rho_{2,1}$ and $\rho_{2,0}>-1$, then for any compactly supported $f \in C_{c}^{\infty}(M)$,

$$
\left\|\Gamma^{\mathbf{h}^{*}}\left(P_{t} f\right)\right\|_{L^{1}} \leq e^{-\alpha t}\left\|\Gamma^{\mathbf{h}^{*}}(f)\right\|_{L^{1}}, \quad \alpha:=\frac{\rho_{2,0} \rho_{1}+\rho_{2,1}}{\rho_{2,0}+1} .
$$

Furthermore, if $\alpha>0$ then $\operatorname{vol}(M)<\infty$.

(b) Assume that the conditions in (a) hold with $\alpha>0$. Then for any $f \in C_{c}^{\infty}(M)$,

$$
\left\|f-f_{M}\right\|_{L^{2}}^{2} \leq \frac{1}{\alpha} \int_{M} \Gamma^{\mathbf{h}^{*}}(f) d \mathrm{vol}
$$

where $f_{M}=\operatorname{vol}(M)^{-1} \int_{M} f d \operatorname{vol}$. As a consequence if $\lambda$ is a non-zero eigenvalue of $L$, then $\alpha \leq-\lambda$.

There are also other inequalities which do not require that $L$ is symmetric with respect to a volume form, see e.g. Part II, Proposition 3.6. 
For the case when both (A) and (B) hold and $L$ satisfies (CD) of Section 3.3 with $\rho_{2}>0$, we can use the results from [8, 6, 7]. In particular, with some extra computation, we can conclude the following.

Corollary 5.3 (Part II, Proposition 5.1, Proposition 5.3). Let $\Delta_{\mathbf{h}}$ is as in Theorem 5.1 (b). Let

$$
\kappa=\frac{1}{2} m_{\mathcal{R}} \rho_{\mathcal{H}}-\mathscr{M}_{\mathcal{H} \mathcal{V}}^{2} .
$$

(a) Assume that $\kappa>0$. Then $M$ is compact, and for any $f \in C^{\infty}(M)$,

$$
\left\|f-f_{M}\right\|_{L^{2}}^{2} \leq \frac{1}{\alpha} \int_{M} \Gamma^{\mathbf{h}^{*}}(f) d \mathrm{vol}
$$

where $f_{M}=\operatorname{vol}(M)^{-1} \int_{M} f d \operatorname{vol}$ and

$$
\alpha:=\left(\frac{2 \kappa}{2 \mathscr{M}_{\mathcal{H} \mathcal{V}}+m_{\mathcal{R}} \sqrt{2 \rho_{\mathcal{H}}+2 \kappa}}\right)^{2} .
$$

(b) Assume that $\kappa \geq 0$ and define

$$
N=\frac{n}{4} \frac{\left(\sqrt{2 \rho_{\mathcal{H}}+\kappa}+\sqrt{\rho_{\mathcal{H}}+\kappa}\right)^{2}}{\kappa}, \quad D=\frac{\sqrt{\left(\kappa+\rho_{\mathcal{H}}\right)\left(\kappa+2 \rho_{\mathcal{H}}\right)}}{\kappa} .
$$

Let $p_{t}(x, y)$ be the heat kernel of $\frac{1}{2} \Delta_{\mathbf{h}}$ with respect to vol. Then

$$
p_{t}(x, x) \leq \frac{1}{t^{N / 2}} p_{1}(x, x)
$$

where $x \in M$ and $0 \leq t \leq 1$. Furthermore, for any $0<t_{0}<t_{1}$, any nonnegative smooth bounded function $f \in C_{b}^{\infty}(M)$ and points $x, y \in M$,

$$
P_{t_{0}} f(x) \leq\left(P_{t_{1}} f\right)(y)\left(\frac{t_{1}}{t_{0}}\right)^{N / 2} \exp \left(D \frac{\mathrm{d}(x, y)^{2}}{2\left(t_{1}-t_{0}\right)}\right) .
$$

If $\kappa=0$, we interpret the quotient $\kappa / \rho_{\mathcal{H}}$ as $m_{\mathcal{R}}^{2} / 2$.

\section{REFERENCES}

[1] A. Agrachev, D. Barilari, and L. Rizzi. Curvature: a variational approach. To appear in: Memoirs AMS

[2] A. Agrachev, U. Boscain, J.-P. Gauthier, and F. Rossi. The intrinsic hypoelliptic Laplacian and its heat kernel on unimodular Lie groups. J. Funct. Anal., 256(8):2621-2655, 2009.

[3] D. Bakry and M. Émery. Diffusions hypercontractives. In Séminaire de probabilités, XIX, 1983/84, volume 1123 of Lecture Notes in Math., pages 177-206. Springer, Berlin, 1985.

[4] D. Bakry and M. Ledoux. Lévy-Gromov's isoperimetric inequality for an infinite-dimensional diffusion generator. Invent. Math., 123(2):259-281, 1996.

[5] D. Barilari and L. Rizzi. Comparison theorems for conjugate points in sub-Riemannian geometry. To appear in: ESAIM Control Optim. Calc. Var.

[6] F. Baudoin and M. Bonnefont. Log-Sobolev inequalities for subelliptic operators satisfying a generalized curvature dimension inequality. J. Funct. Anal., 262(6):2646-2676, 2012.

[7] F. Baudoin, M. Bonnefont, and N. Garofalo. A sub-Riemannian curvature-dimension inequality, volume doubling property and the Poincaré inequality. Math. Ann., 358(3-4):833-860, 2014.

[8] F. Baudoin and N. Garofalo. Curvature-dimension inequalities and Ricci lower bounds for sub-Riemannian manifolds with transverse symmetries. To appear in: Journal of the EMS.

[9] F. Baudoin, B. Kim, and J. Wang. Transverse Weitzenböck formulas and curvature dimension inequalities on Riemannian foliations with totally geodesic leaves. ArXiv e-prints: 1408.0548 , Aug. 2014.

[10] F. Baudoin and J. Wang. Curvature dimension inequalities and subelliptic heat kernel gradient bounds on contact manifolds. Potential Anal., 40(2):163-193, 2014. 
[11] R. Bott. On a topological obstruction to integrability. In Global Analysis (Proc. Sympos. Pure Math., Vol. XVI, Berkeley, Calif., 1968), pages 127-131. Amer. Math. Soc., Providence, R.I., 1970.

[12] W.-L. Chow. Über Systeme von linearen partiellen Differentialgleichungen erster Ordnung. Math. Ann., 117:98-105, 1939.

[13] M. Émery. Stochastic calculus in manifolds. Universitext. Springer-Verlag, Berlin, 1989. With an appendix by P.-A. Meyer.

[14] R. K. Hladky. Bounds for the first eigenvalue of the horizontal Laplacian in positively curved sub-Riemannian manifolds. Geom. Dedicata, 164:155-177, 2013.

[15] L. Hörmander. Hypoelliptic second order differential equations. Acta Math., 119:147-171, 1967.

[16] E. P. Hsu. Stochastic analysis on manifolds, volume 38 of Graduate Studies in Mathematics. American Mathematical Society, Providence, RI, 2002.

[17] I. Kolář, P. W. Michor, and J. Slovák. Natural operations in differential geometry. SpringerVerlag, Berlin, 1993.

[18] C. Li and I. Zelenko. Jacobi equations and comparison theorems for corank 1 sub-Riemannian structures with symmetries. J. Geom. Phys., 61(4):781-807, 2011.

[19] P.-A. Meyer. Géométrie stochastique sans larmes. In Seminar on Probability, XV (Univ. Strasbourg, Strasbourg, 1979/1980) (French), volume 850 of Lecture Notes in Math., pages 44-102. Springer, Berlin-New York, 1981.

[20] R. Montgomery. A tour of subriemannian geometries, their geodesics and applications, volume 91 of Mathematical Surveys and Monographs. American Mathematical Society, Providence, RI, 2002.

[21] P. K. Rashevskii. On joining any two points of a completely nonholonomic space by an admissible line. Math. Ann., 3:83-94, 1938.

[22] B. L. Reinhart. Foliated manifolds with bundle-like metrics. Ann. of Math. (2), 69:119-132, 1959.

[23] I. Shigekawa. On stochastic horizontal lifts. Z. Wahrsch. Verw. Gebiete, 59(2):211-221, 1982.

[24] R. S. Strichartz. Sub-Riemannian geometry. J. Differential Geom., 24(2):221-263, 1986.

[25] K.-T. Sturm. Analysis on local Dirichlet spaces. I. Recurrence, conservativeness and $L^{p_{-}}$ Liouville properties. J. Reine Angew. Math., 456:173-196, 1994.

[26] F.-Y. Wang. Equivalence of dimension-free Harnack inequality and curvature condition. Integral Equations Operator Theory, 48(4):547-552, 2004.

[27] I. Zelenko and C. Li. Parametrized curves in Lagrange Grassmannians. C. R. Math. Acad. Sci. Paris, 345(11):647-652, 2007.

Mathematics Research Unit, University of Luxembourg, 6 Rue Richard CoudenhoveKALERGi, L-1359 LuXEMBourg

E-mail address: erlend.grong@uni.lu

Mathematics Research Unit, University of Luxembourg, 6 Rue Richard CoudenhoveKalergi, L-1359 LuXembourG

E-mail address: anton.thalmaier@uni.lu 